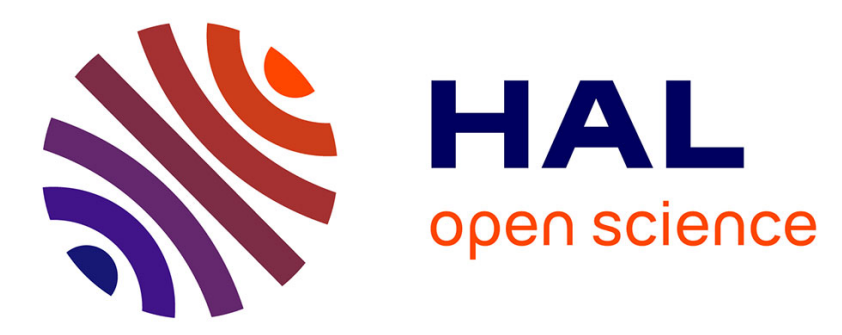

\title{
Gamma regularization based reconstruction for low dose CT
}

\author{
Junfeng Zhang, Yang Chen, Yining Hu, Limin Luo, Huazhong Shu, Bicao Li, \\ Jin Liu, Jean-Louis Coatrieux
}

\section{> To cite this version:}

Junfeng Zhang, Yang Chen, Yining Hu, Limin Luo, Huazhong Shu, et al.. Gamma regularization based reconstruction for low dose CT. Magnetic Resonance Materials in Physics, Biology and Medicine, 2015, 60 (17), pp.6901-6921. 10.1088/0031-9155/60/17/6901 . hal-01222764

HAL Id: hal-01222764

https://hal.science/hal-01222764

Submitted on 30 Oct 2015

HAL is a multi-disciplinary open access archive for the deposit and dissemination of scientific research documents, whether they are published or not. The documents may come from teaching and research institutions in France or abroad, or from public or private research centers.
L'archive ouverte pluridisciplinaire HAL, est destinée au dépôt et à la diffusion de documents scientifiques de niveau recherche, publiés ou non, émanant des établissements d'enseignement et de recherche français ou étrangers, des laboratoires publics ou privés. 


\title{
Gamma Regularization Based Reconstruction for Low Dose CT
}

\author{
Junfeng Zhang ${ }^{1}$, Yang Chen ${ }^{1,2,3}$, Yining $\mathrm{Hu}^{1,2,3}$, Limin $\mathrm{Luo}^{1,2,3}$, Huazhong Shu ${ }^{1,2,3}$, Bicao $\mathrm{Li}^{1,2,3}$, Jin
} Liu $^{1,2,3}$, Jean-Louis Coatrieux ${ }^{3,4,5}$

1. Laboratory of Image Science and Technology, Southeast University, Nanjing, China;

2. Key Laboratory of Computer Network and Information Integration (Southeast University), Ministry of Education, China

3. Centre de Recherche en Information Biomédicale Sino-Francais (LIA CRIBs), Rennes, France;

4. INSERM, U1099, Rennes, F-35000, France;

5. Université de Rennes 1, LTSI, Rennes, F-35000, France.

\section{E-mail: chenyang.list@seu.edu.cn}

\begin{abstract}
Reducing the radiation in computerized tomography is today a major concern in radiology. Low dose computerized tomography (LDCT) offers a sound way to deal with this problem. However, more severe noise in the reconstructed CT images is observed under low dose scan protocols (e.g. lowered tube current or voltage values). In this paper we propose a Gamma regularization based algorithm for LDCT image reconstruction. This solution provides a good balance between the regularizations based on $l_{0}$-norm and $l_{1}$-norm. We evaluate the proposed approach using the projection data from simulated phantoms and scanned Catphan phantoms. Qualitative and quantitative results show that the Gamma regularization based reconstruction can perform better in both edge-preserving and noise suppression when compared with other regularizations using integer norms.
\end{abstract}

Key words: Low dose computerized tomography (LDCT), Gamma regularization, weighted least square (WLS)

\section{Introduction}

CT examination is undoubtedly an effective and reliable medical tool in providing anatomical and pathological information for clinical diagnosis. However, it has been shown that the inherent radiation of CT scanners can induce cancer and other diseases (Brenner et al 2001, Brenner et al 2007, de González and Darby 2004). With reduced radiation harm to patients, low dose computerized tomography reconstruction has attracted more and more research attention. However, when the dose is reduced (e.g. by lowering the tube current or voltage values), the images reconstructed using for instance the Filtered Back Projection (FBP) suffer from increased noise and artifacts. Diagnostic mistakes might be induced in this case. In the past decades, a number of attempts have been carried out to overcome this shortcoming and to provide clinically acceptable LDCT images (Lange and Carson 1984, Hsieh 1998, Lu et al 2001, Li et al 2004, Sidky et al 2006, Wang et al 2006, Hsieh 2009, Yu and Wang 2010, Hu et al 2011, Xu et al 2012, Chen et al 2013, Liu et al 2014, Niu et al 2014). Among all the low dose strategies, lowering the tube current values (milliampere $(\mathrm{mA})$ or milliampere second $(\mathrm{mAs}))$ or the voltage values (kilovolt $(\mathrm{KV})$ ) is the most straightforward and pragmatic solution. But in such case, the quality of projection data is degraded due to a decrease in photon number and an amplified noise turbulence (Hsieh 1998, Li et al 2004, Lu et al 2001). By better modeling the projection data and the imaging geometry, statistical reconstruction algorithms have been shown more effective than FBP in the LDCT (Lange and Carson 1984, Elbakri and Fessler 2002, Li et al 2004, Wang et al 2006).

Another path has been recently open by compressed sensing (CS) with already wide applications in medical image processing, e.g. magnetic resonance imaging (MRI), bioluminescence tomography, optical coherence tomography (Candes et al 2006a, Candes et al 2006b, Donoho 2006, Lustig et al 2007, Lu et al 2009, Fang et al 2013). Studies on CS theory unveil the possibility of recovering sparse signals when the requirement specified by the Nyquist sampling theorem cannot be satisfied (Candes et al 2006a). Although the restricted isometry property (RIP) condition is not often satisfied in practice, CS-based reconstruction can yield more satisfactory results than the traditional FBP algorithm in CT reconstruction ( $\mathrm{Yu}$ and Wang 2010). It is notable that the CS based dictionary learning methods have 
been proved to be rather effective in improving LDCT image quality (Chen et al 2014, Chen et al 2013, Lu et al 2012, Xu et al 2012). The core of CS is based on the sparse assumption of some features in the reconstructed images. It is well-known that the $l_{0}$-norm prior can provide a sparser representation than the $l_{1}$-norm and $l_{2}$-norm priors (Gonzalez et al 2004, Rudin et al 1992). But the application of $l_{0}$-norm prior in reconstruction is often a NP hard problem, the $l_{0}$-norm being a non-convex function in discontinuous form (Candes et al 2006a).

Functions with fixed integer norms are often used in building regularized function for LDCT, e.g. the $l_{1}$-norm and $l_{2}$-norm based regularizations. Then, a natural question would be whether better results can be obtained if we use regularization forms with fractional norm between $l_{0}$-norm and $l_{1}$-norm. In this paper, we propose a Gamma regularization with tunable fractional order norm for LDCT reconstruction. This Gamma regularization allows a flexible regularization modulation and can achieve a good balance between the regularizations based on $l_{0}$ norm and $l_{1}$ norm. The rest of this paper is organized as follows. In section 2, we review the statistical reconstruction model and then detail the proposed Gamma regularization. The reconstruction algorithm using the proposed Gamma regularization is given in section 3. Section 4 includes the experiments conducted on the projection data from both simulated phantoms and scanned Catphan phantoms. The experiment results show that the proposed Gamma regularization leads to better reconstructions than those using integer norm regularizations. Concluding remarks and future work plan are sketched in section 5.

\section{Method}

\subsection{Statistical reconstruction modeling}

The standard CT reconstruction problem can be considered as an inverse problem formulated by Eq. (1):

$$
G f=y
$$

where $f$ denotes the target image of discrete attenuation coefficients, and $y$ represents the calibrated and log-transformed projection data as measurements. $G$ is the system matrix operator reflecting the specific geometry of a given CT imaging system, which can be calculated via techniques like "pixel-driven" (Peters et al 1981, Joseph et al 1982), "ray-driven" (Siddon 1985, Zhuang et al 1994) or "distance-driven" (Man and Basu 2004).

As pointed in Lu et al (2001) and Li et al (2004), for low dose CT with low tube currents, the calibrated log-transformed projection data is degraded by additive Gaussian noise, and the relation between the detected projection data $\tilde{y}$ and the true projection data $y$ for each channel $i$ is:

$$
\begin{aligned}
\tilde{y}_{i}=y_{i}+\mu_{i} & =(G f)_{i}+\operatorname{Gaussian}\left(0, \sigma_{i}^{2}\right) \\
\sigma_{i}^{2} & =h \times \exp \left(\frac{y_{i}}{T}\right)
\end{aligned}
$$

Here, Gaussian $\left(0, \sigma_{i}^{2}\right)$ denotes the Gaussian noise term $\mu_{i}$ for each channel $i$ with zero mean and the variance specified by Eq. (3). $\sigma_{i}^{2}$ is the variance of projection measurement $y_{i}$ at channel $i$. $T$ and $h$ are object-independent parameters completely determined by the system and can be determined by fitting a large amount of projection data from repeated scanning. Through maximizing the likelihood estimation, we can reconstruct image $f$ by minimizing the weighted least square (WLS) model:

$$
f=\underset{f}{\arg \min }\left\{\Phi(f)=\frac{1}{2} W\|G f-\tilde{y}\|^{2}\right\}
$$

Where $W$ is a diagonal matrix, with the ith entry $1 / \sigma_{i}^{2}$. Solving $f$ via Eq. (4) is in fact an ill-posed problem because the rank of the system matrix $G$ is often lower than the rank of $f$, which results in infinite possible solutions (Tikhonov and Arsenin 1997). Some prior knowledge on image $f$ can be used to overcome this ill-posed problem by adding a regularization term $\Psi(f)$ into the Eq. (4), which leads to the following regularization model Eq. (5):

$$
f=\underset{f}{\arg \min }\left\{\Phi(f)=\frac{1}{2} W\|G f-\tilde{y}\|^{2}+\lambda \Psi(f)\right\}
$$

Here, $\lambda$ is a positive regularization parameter modulating the trade-off between the fidelity term and the regularization term. The prior knowledge can be introduced in $\Psi(f)$ using different functions of 
gradients. We can for instance define the anisotropic regularization $\Psi_{a}(f)$ and isotropic regularization $\Psi_{i}(f)$ (Teboulle, 2009), (Guo and Yin, 2012):

$$
\begin{array}{r}
\Psi(f)=\Psi_{a}(f)=\sum_{i=1}^{m} \sum_{j=1}^{n}\left(\psi\left(\left|\nabla f_{i, j}^{v}\right|\right)+\psi\left(\left|\nabla f_{i, j}^{h}\right|\right)\right) \\
\Psi(f)=\Psi_{i}(f)=\sum_{i=1}^{m} \sum_{j=1}^{n} \psi\left(\sqrt{\left(\nabla f_{i, j}^{v}\right)^{2}+\left(\nabla f_{i, j}^{h}\right)^{2}}\right)
\end{array}
$$

In Eq. (6) and Eq. (7), $\psi$ is the regularization function acting on the gradient term, and $f_{i, j}$ represents each 2-D pixel in the reconstructed 2D image with index $i=1, \mathrm{~K} m$ and $j=1, \mathrm{~K} n$. Here, $\nabla f_{i, j}$ takes the form $\left(\nabla f_{i, j}^{v}, \nabla f_{i, j}^{h}\right)$ including the vertical and horizontal gradients $\nabla f_{i, j}^{v}$ and $\nabla f_{i, j}^{h}$ :

$$
\nabla f_{i, j}^{v}=\left\{\begin{array}{ll}
f_{i+1, j}-f_{i, j} & i<m \\
0 & i=m
\end{array}, \quad \nabla f_{i, j}^{h}=\left\{\begin{array}{ll}
f_{i, j+1}-f_{i, j} & j<n \\
0 & j=n
\end{array}, \quad i=1, \mathrm{~K} m, j=1, \mathrm{~K}, n\right.\right.
$$

Different regularization function $\psi$ can be used in building $\Psi(f)$. Using $\psi(x)=x^{2}$ in the models expressed by Eq. (6) or Eq. (7), we obtain the $l_{2}$-norm regularization based WLS reconstruction model ( $l_{2}$-WLS) (Gonzalez et al 2004):

$$
f=\arg \min _{f}\left\{\Phi(f)=\frac{1}{2} W\|G f-\tilde{y}\|_{2}^{2}+\lambda \sum_{i=1}^{m} \sum_{j=1}^{n}\left(\left|\nabla f_{i, j}^{v}\right|^{2}+\left|\nabla f_{i, j}^{h}\right|^{2}\right)\right\}
$$

If setting $\psi(x)=x$ in Eq. (6) for the isotropic model, we obtain the $l_{1}$-norm anisotropic regularization WLS reconstruction model ( $l_{1 a}$-WLS) (Beck and Teboulle 2009, Guo and Yin 2012):

$$
f=\arg \min _{f}\left\{\Phi(f)=\frac{1}{2} W\|G f-\tilde{y}\|_{2}^{2}+\lambda\left(\sum_{i=1}^{m} \sum_{j=1}^{n}\left|\nabla f_{i, j}^{h}\right|+\sum_{i=1}^{m} \sum_{i=1}^{n} \mid \nabla f_{i, j}^{v}\right)\right\}
$$

Similarly, with $\psi(x)=x$ in the isotropic model in Eq. (7), the $l_{1}$-norm isotropic regularization WLS reconstruction model ( $l_{1 i}$-WLS) (Rudin et al1992) can be derived:

$$
f=\arg \min _{f}\left\{\Phi(f)=\frac{1}{2} W\|G f-\tilde{y}\|_{2}^{2}+\lambda \sum_{i=1}^{m} \sum_{j=1}^{n} \sqrt{\left|\nabla f_{i, j}^{h}\right|^{2}+\left|\nabla f_{i, j}^{v}\right|^{2}}\right\}
$$

\subsection{The proposed Gamma regularization WLS model $\left(\Gamma_{a}-W L S\right.$ and $\left.\Gamma_{i}-W L S\right)$}

Through calculus transform, we can easily rewrite the WLS reconstruction model with the $l_{2}$-norm regularization Eq. (9) into Eq. (12):

$$
f=\arg \min _{f}\left\{\Phi(f)=\frac{1}{2} W\|G f-\tilde{y}\|_{2}^{2}+\lambda \sum_{i=1}^{m} \sum_{j=1}^{n}\left(\int_{0}^{\left|\nabla f_{i, j}^{v}\right|} 2 t d t+\int_{0}^{\left|\nabla f_{i, j}^{h}\right|} 2 t d t\right)\right\}
$$

Similarly, Eq. (10) and Eq. (11) can be respectively rewritten by Eq. (13) and Eq. (14):

$$
\begin{gathered}
f=\arg \min _{f}\left\{\Phi(f)=\frac{1}{2} W \| G f-\left.\tilde{y}\right|_{2} ^{2}+\lambda\left(\sum_{i=1}^{m} \sum_{j=1}^{n} \int_{0}^{\left|\nabla f_{i, j}^{h}\right|} d t+\sum_{i=1}^{m} \sum_{i=1}^{n} \int_{0}^{\left|\nabla f_{i, j}^{n}\right|} d t\right)\right\} \\
f=\arg \min _{f}\left\{\Phi(f)=\frac{1}{2} W\|G f-\tilde{y}\|_{2}^{2}+\lambda \sum_{i=1}^{m} \sum_{j=1}^{n} \int_{0}^{\sqrt{\left.\left|\nabla f_{i, j}^{h}\right|^{2}|+| f_{i, j}^{\prime}\right|^{2}}} d t\right\}
\end{gathered}
$$

Based on Eq. (12)-(14), a generalized reconstruction model Eq. (15) can be obtained by introducing a integrand function $\phi(t)$ :

$$
f=\underset{f}{\arg \min }\left\{\Phi(f)=\frac{1}{2} W\|G f-\tilde{y}\|_{2}^{2}+\lambda \sum_{i=1}^{m} \sum_{j=1}^{n}\left(\int_{0}^{\left|\nabla f_{i, j}^{n}\right|} \phi(t) d t+\int_{0}^{\left|\nabla f_{i, j}^{n}\right|} \phi(t) d t\right)\right\}
$$

where $\phi(t)$ denotes the general kernel function with positive constraint $(\phi(t) \geq 0)$. Note Eq. (15) will become Eq. (9) or Eq. (10) when $\phi(t)=2 t$ or 1 .

We propose a new kernel function $\phi_{\Gamma}(t ; \alpha, \beta)$ based on the Gamma probability density function (PDF) in the forms of Eq. (16) and Eq. (17) (Hogg and Craig 1978):

$$
\phi_{\Gamma}(t ; \alpha, \beta)=\frac{t^{\alpha-1} \beta^{\alpha} e^{-\beta t}}{\Gamma(\alpha)}
$$




$$
\Gamma(\alpha)=\int_{0}^{\infty} \frac{t^{\alpha-1}}{e^{t}} d t
$$

In Eq. (16), $\alpha$ and $\beta$ denote the shape parameter and the scale parameter, respectively. The Gamma-distributed random variable $t$ in Eq. (16) has expectation $E=\alpha / \beta$ and variance $V=$ $\alpha / \beta^{2}$, respectively (Hogg and Craig 1978). Then, the Gamma regularization function $\psi_{\Gamma}(x ; \alpha, \beta)$ can be defined as:

$$
\psi_{\Gamma}(x ; \alpha, \beta)=\int_{0}^{x} \frac{t^{\alpha-1} \beta^{\alpha} e^{-\beta t}}{\Gamma(\alpha)} d t
$$

Here, $\psi_{\Gamma}(x ; \alpha, \beta)$ is in fact the cumulative distribution function (CDF) of the Gamma distributed random variable $t$ with shape parameter $\alpha$ and scale parameter $\beta$ (Hogg and Craig 1978). Fig. 1 depicts the regularization functions for $l_{2}$-norm regularization, $l_{1}$-norm regularization, $l_{0}$-norm regularization, and the proposed Gamma regularization function $\psi_{\Gamma}(x ; \alpha, \beta)$ with different shape parameter $\alpha \in\{1.0,1.2\}$ and scale parameter $\beta \in\{2,4,6,8\}$. We can see in Fig. 1 that the Gamma regularization functions can be modulated by selecting different values of $\alpha$ and $\beta$ to get a flexible trade-off between $l_{1}$-norm regularization and $l_{0}$-norm regularization.
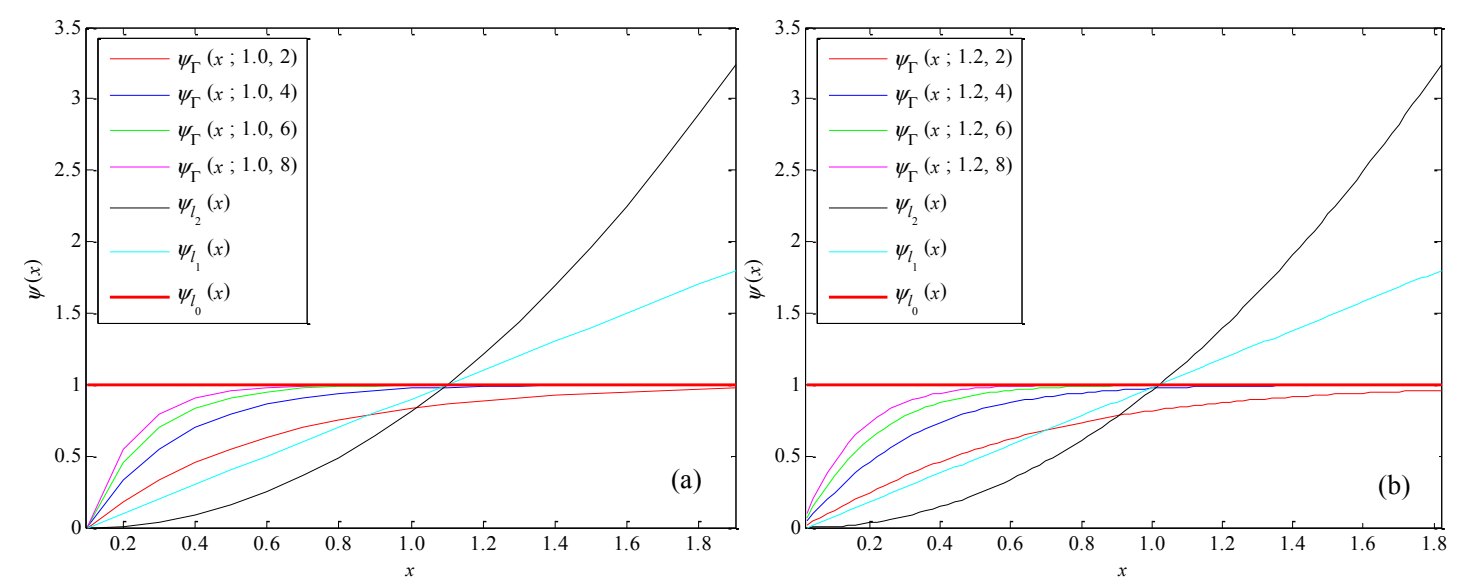

Fig. 1: Regularization functions $\left(\psi_{l_{2}}(x), \psi_{l_{1}}(x), \psi_{l_{0}}(x), \psi_{\Gamma}(x ; \alpha, \beta)\right)$ for $l_{2}$-norm, $l_{1}$-norm, $l_{0}$-norm, and Gamma regularizations (with different shape parameter $\alpha$ and scale parameter $\beta$ in $\psi_{\Gamma}(x ; \alpha, \beta)$ ).

Consequently, the cost function for the reconstruction with anisotropic Gamma regularization ( $\Gamma_{a}$-WLS) can be given by Eq. (19):

$$
f=\underset{f}{\arg \min }\left\{\Phi(f)=\frac{1}{2} W\|G f-\tilde{y}\|_{2}^{2}+\lambda \Psi_{\Gamma_{a}}(f)\right\}
$$

where

$$
\Psi_{\Gamma_{a}}(f)=\sum_{i=1}^{m} \sum_{j=1}^{n} \int_{0}^{\nabla f_{i, j}^{v}} \frac{t^{\alpha-1} \beta^{\alpha} e^{-\beta t}}{\Gamma(\alpha)} d t+\sum_{i=1}^{m} \sum_{j=1}^{n} \int_{0}^{\left|\nabla f_{i, j}^{h}\right|} \frac{t^{\alpha-1} \beta^{\alpha} e^{-\beta t}}{\Gamma(\alpha)} d t
$$

Accordingly, the cost function for isotropic Gamma regularization $\left(\Gamma_{i}-\mathrm{WLS}\right)$ is defined as :

$$
f=\underset{f}{\arg \min }\left\{\Phi(f)=\frac{1}{2} W\|G f-\tilde{y}\|_{2}^{2}+\lambda \Psi_{\Gamma_{i}}(f)\right\}
$$

where

$$
\Psi_{\Gamma_{i}}(f)=\sum_{i=1}^{m} \sum_{j=1}^{n} \int_{0}^{\sqrt{\left|\nabla f_{i, j}^{h}\right|^{2}+\left|\nabla f_{i, j}^{v}\right|^{2}}} \frac{t^{\alpha-1} \beta^{\alpha} e^{-\beta t}}{\Gamma(\alpha)} d t
$$

\section{Optimization algorithm}

3.1 Algorithm description

The conjugate gradient method (CG) (Boyd S and Vandenberghe 2004) was chosen to solve the reconstruction problems in Eq. (19) and Eq. (21). The CG algorithm is an improved steepest descent 
algorithm, with the descent direction determined by the current descent direction as well as the previous search direction (Fletcher and Reeves 1964). Since the optimization of Eq. (19) is similar, we only derive the algorithm solving Eq. (21). We first define the partial derivative $[\nabla \Phi(f)]_{i, j}$ of the Gamma regularization term in Eq. (21) with respect to each image point $f_{i, j}$ :

$$
[\nabla \Phi(f)]_{i, j}=\left(G^{T} W(G f-\tilde{y})\right)_{i, j}+\lambda \frac{\partial}{\partial f_{i, j}}\left(\Psi_{\Gamma_{i}}(f)\right)
$$

The partial derivative of the regularization term with respect to pixel $f_{i, j}$ is:

$$
\begin{aligned}
\frac{\partial}{\partial f_{i, j}}\left(\Psi_{\Gamma_{i}}(f)\right)= & -\frac{\left(\left(f_{i+1, j}-f_{i, j}\right)^{2}+\left(f_{i, j+1}-f_{i, j}\right)^{2}\right)^{\frac{\alpha}{2}-1} \beta^{\alpha} e^{-\beta\left(\left(f_{i+1, j}-f_{i, j}\right)^{2}+\left(f_{i, j+1}-f_{i, j}\right)^{2}\right)^{\frac{1}{2}}}}{\Gamma(\alpha)}\left(f_{i+1, j}-f_{i, j}\right) \\
& -\frac{\left(\left(f_{i+1, j}-f_{i, j}\right)^{2}+\left(f_{i, j+1}-f_{i, j}\right)^{2}\right)^{\frac{\alpha}{2}-1} \beta^{\alpha} e^{-\beta\left(\left(f_{i+1, j}-f_{i, j}\right)^{2}+\left(f_{i, j+1}-f_{i, j}\right)^{2}\right)^{\frac{1}{2}}}}{\Gamma(\alpha)}\left(f_{i, j+1}-f_{i, j}\right) \\
& +\frac{\left(\left(f_{i, j}-f_{i-1, j}\right)^{2}+\left(f_{i-1, j+1}-f_{i-1, j}\right)^{2}\right)^{\frac{\alpha}{2}-1} \beta^{\alpha} e^{-\beta\left(\left(f_{i, j}-f_{i-1, j}\right)^{2}+\left(f_{i-1, j+1}-f_{i-1, j}\right)^{2}\right)^{\frac{1}{2}}}}{\Gamma(\alpha)}\left(f_{i, j}-f_{i-1, j}\right) \\
+ & \frac{\left(\left(f_{i+1, j-1}-f_{i, j-1}\right)^{2}+\left(f_{i, j}-f_{i, j-1}\right)^{2}\right)^{\frac{\alpha}{2}-1} \beta^{\alpha} e^{-\beta\left(\left(f_{i+1, j-1}-f_{i, j-1}\right)^{2}+\left(f_{i, j}-f_{i, j-1}\right)^{2}\right)^{\frac{1}{2}}}}{\Gamma(\alpha)}\left(f_{i, j}-f_{i, j-1}\right)
\end{aligned}
$$

The Gamma regularization term Eq. (22) can be approximated by Eq. (25):

$$
\Psi_{\Gamma_{i}}(f) \approx \sum_{i=1}^{m} \sum_{j=1}^{n} \int_{0}^{\left(\left|\nabla f_{i, j}^{\mathrm{h}}\right|^{2}+\left|\nabla f_{i, j}^{y}\right|^{2}+\varepsilon\right)^{\frac{1}{2}}} \frac{t^{\alpha-1} \beta^{\alpha} e^{-\beta t}}{\Gamma(\alpha)} d t
$$

Here, $\varepsilon$ is a small constant used to avoid the zero denominator in Eq. (24) (when $\alpha<2$ ). Then, the corresponding partial derivative of the regularization term Eq. (25) with respect to pixel $f_{i, j}$ is:

$$
\begin{aligned}
\frac{\partial}{\partial f_{i, j}}\left(\Psi_{\Gamma_{i}}(f)\right) & =-\frac{\left(\left(f_{i+1, j}-f_{i, j}\right)^{2}+\left(f_{i, j+1}-f_{i, j}\right)^{2}+\varepsilon\right)^{\frac{\alpha}{2}-1} \beta^{\alpha} e^{-\beta\left(\left(f_{i+1, j}-f_{i, j}\right)^{2}+\left(f_{i, j+1}-f_{i, j}\right)^{2}+\varepsilon\right)^{\frac{1}{2}}}}{\Gamma(\alpha)}\left(f_{i+1, j}-f_{i, j}\right) \\
& -\frac{\left(\left(f_{i+1, j}-f_{i, j}\right)^{2}+\left(f_{i, j+1}-f_{i, j}\right)^{2}+\varepsilon\right)^{\frac{\alpha}{2}-1} \beta^{\alpha} e^{-\beta\left(\left(f_{i+1, j}-f_{i, j}\right)^{2}+\left(f_{i, j+1}-f_{i, j}\right)^{2}+\varepsilon\right)^{\frac{1}{2}}}}{\Gamma(\alpha)}\left(f_{i, j+1}-f_{i, j}\right) \\
+ & \frac{\left(\left(f_{i+1, j-1}-f_{i, j-1}\right)^{2}+\left(f_{i, j}-f_{i, j-1}\right)^{2}+\varepsilon\right)^{\frac{\alpha}{2}-1} \beta^{\alpha} e^{-\beta\left(\left(f_{i+1, j-1}-f_{i, j-1}\right)^{2}+\left(f_{i, j}-f_{i, j-1}\right)^{2}+\varepsilon\right)^{\frac{1}{2}}}}{\Gamma(\alpha)}\left(f_{i, j}-f_{i, j-1}\right) \\
+ & \frac{\left(\left(f_{i, j}-f_{i-1, j}\right)^{2}+\left(f_{i-1, j+1}-f_{i-1, j}\right)^{2}+\varepsilon\right)^{\frac{\alpha}{2}-1} \beta^{\alpha} e^{-\beta\left(\left(f_{i, j}-f_{i-1, j}\right)^{2}+\left(f_{i-1, j+1}-f_{i-1, j}\right)^{2}+\varepsilon\right)^{\frac{1}{2}}}}{\Gamma(\alpha)}\left(f_{i, j}-f_{i-1, j}\right)
\end{aligned}
$$

The overall solution is outlined in Algorithm 1 below. We applied the strategy of backtracking line search to improve its convergence. In this strategy, the iteration step $\tau$ is recursively selected to determine the maximum step along a given search direction by means of a backtracking line search with the Armijo-Goldstein condition (Armijo 1966, Frank and Wolfe 1956):

$$
\Phi(f+\tau d)<\Phi(f)+\eta \tau\left(\frac{\partial}{\partial f} \Phi(f)\right)^{T} d
$$

where $\eta \in[0,0.5], d$ is the descent direction and the superscript " $T$ " denotes the matrix transpose operator. The basic idea of the backtracking line search is to recursively decrease the step $\tau$ from a large preset value until a step $\tau$ is found to satisfy the constraint of Eq. (27). In the Algorithm 1, the 
iteration stopping is jointly controlled by the iteration update limit $\varepsilon_{0}$ and the maximum iteration number $N_{0}$.

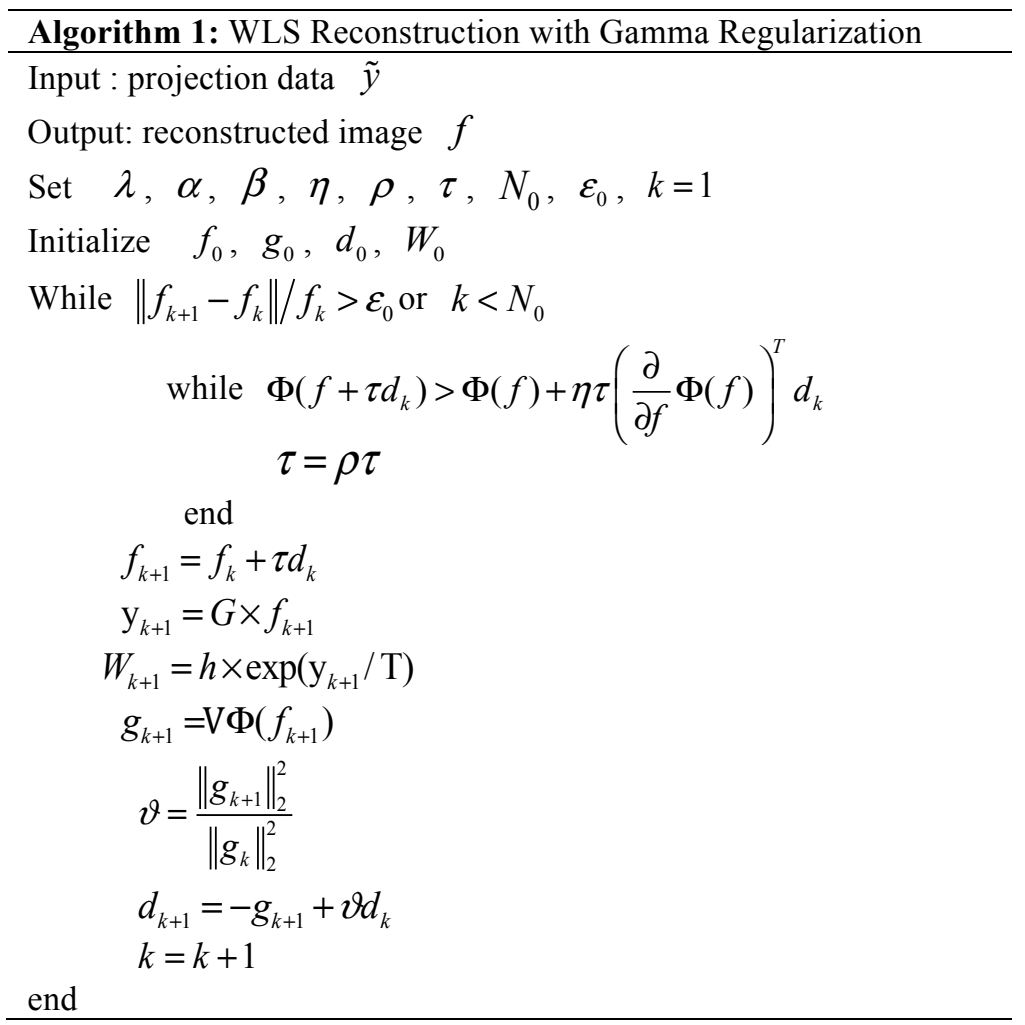

From the above algorithm outline, we can easily obtain the following relation:

$$
\begin{gathered}
\Phi\left(f_{k+1}\right)=\frac{1}{2} W_{k+1}\left\|G f_{k+1}-\tilde{y}\right\|^{2}+\lambda\left(\psi_{\bullet_{i}}\left(\sqrt{\left|\nabla f_{i, j}^{\mathrm{h}}\right|^{2}+\left|\nabla f_{i, j}^{v}\right|^{2}}\right)\right) \\
\leq \frac{1}{2} W_{k}|| G f_{k}-\tilde{y} \|^{2}+\lambda\left(\psi_{\bullet_{i}}\left(\sqrt{\left|\nabla f_{i, j}^{\mathrm{h}}\right|^{2}+\left|\nabla f_{i, j}^{v}\right|^{2}}\right)\right)=\Phi\left(f_{k}\right)
\end{gathered}
$$

Where $f_{k}$ denotes the $k^{\text {th }}$ iterated image in reconstruction. This analysis shows that a monotonic decrement of the cost function can be ensured over iterations for the Algorithm 1, which means that a local minimum can be obtained for the proposed reconstruction algorithm.

\subsection{Gamma parameters setting}

The regularization function shown in Eq. (18) monotonically increases with respect to the image gradients and the approximation (to 1) goes stable as gradient increases. The inherent assumption is that the regularization effect should decrease as the gradient values increase because large gradients are often related to image edges, and small gradients often come from noise and artifacts. The shape parameter $\alpha$ and the scale parameter $\beta$ jointly determine the regularization function in Eq. (18), and are crucial to the performance of the proposed reconstruction model. Fig. 2 (a) depicts the regularization function values for different $\beta$ when $\alpha$ is fixed to 1.2, and Fig. 2 (b) the values of the regularization function Eq. (18) for different $\alpha$ in the case of fixed $\beta$. From Fig. 2 (a) and Fig. 2 (b), we can see that the regularization function approximates more closely to the $l_{0}$ norm as $\beta$ increases or $\alpha$ decreases. This observation means that the parameters $\alpha$ and $\beta$ affect the approximation to $l_{0}$ norm in opposite ways. Fig. 2 (c) and (d) illustrate the regularization functions when $\alpha \in\{1.0,1.1,1.2,1.3,1.4,1.5\}$ and the ratio $E$ of $\alpha$ and $\beta$ is fixed to 0.15 and 0.2 , respectively. We can see in Fig. 2 (c) and (d) that the regularization functions take similar shapes for a fixed $E$ value when $\alpha$ is varying. The plots in Fig. 2 (e) also show that the regularization function becomes closer to a binary function when the parameter $\alpha$ increases to 150 and the $E$ is fixed (the 
$\beta$ can be deterministically calculated as $\alpha / E$ ). Considering that a binary regularization function cannot reflect the spatially varying gradients in CT images, the $\alpha$ value is selected within the range $[1.0,1.5]$ in this study.

The plots in Fig. 2 (c) and (d) also show that we can modulate the shapes of regularization functions (or its approximation to 1 ) by selecting a suitable ratio $E$. The Eq. (29) can be derived in order to depict the relation between the regularization function and the ratio $E$ :

$$
\forall \beta, \quad \psi_{\Gamma}(x=5 E ; \alpha, \beta)=\psi_{\Gamma}(5 E ; \alpha, \beta)=\int_{0}^{5 E} \frac{t^{\alpha-1} \beta^{\alpha} e^{-\beta t}}{\Gamma(\alpha)} d t \geq 0.99 \quad \text { s.t. } \alpha \geq 1
$$

The proof of Eq. (29) is provided below.

Proof: by simply transforming the regularization function in Eq. (29), we can get:

$$
\forall \beta, \quad \psi_{\Gamma}(5 E ; \alpha, \beta)=\int_{0}^{5 E} \frac{t^{\alpha-1} \beta^{\alpha} e^{-\beta t}}{\Gamma(\alpha)} d t=\int_{0}^{5 \beta E} \frac{t^{\alpha-1} e^{-t}}{\Gamma(\alpha)} d t=\int_{0}^{5 \alpha} \frac{t^{\alpha-1} e^{-t}}{\Gamma(\alpha)} d t \quad \text { s.t. } \alpha \geq 1
$$

Taking the derivative with respect to the variable $\alpha$, we get:

$$
\forall \beta, \frac{\partial}{\partial \alpha}\left(\psi_{\Gamma}(5 E ; \alpha, \beta)\right)=\frac{5 t^{\alpha-1} e^{-t}}{\Gamma(\alpha)}>0 \text { s.t. } \alpha \geq 1
$$

Eq. (31) implies that the regularization function in Eq. (30) is a monotonically increasing function with respect to $\alpha$ and we have:

$$
\forall \beta, \quad \psi_{\Gamma}(x>5 E ; \alpha, \beta)>\psi_{\Gamma}(5 E ; \alpha, \beta)=\int_{0}^{5 \alpha} \frac{t^{\alpha-1} e^{-t}}{\Gamma(\alpha)} d t \geq \int_{0}^{5} \frac{e^{-t}}{\Gamma(1)} d t=\psi_{\Gamma}(5 E ; 1, \beta) \geq 0.99 \text { s.t. } \alpha \geq 1
$$

Eq. (32) shows that the gradients larger than $5 E \quad(x>5 E)$ will cause nearly constant value 1 with derivatives near to zero for the regularization function, and should be considered as image edges to be preserved in the reconstructed image. In the present study, we set the shape parameter $\alpha$ to 1.2, and the value of $5 E$ to $25 \%$ quantile of the gradient values (R. Hyndman and Y. Fan 1996). Then the scale parameter $\beta$ can thus be simply calculated as $\alpha / E$. This strategy of parameter setting was found robust in this study. In practical situation, the image to be reconstructed is unavailable, and we just use the FBP reconstructed image to calculate the $25 \%$ quantile of the gradient amplitudes.
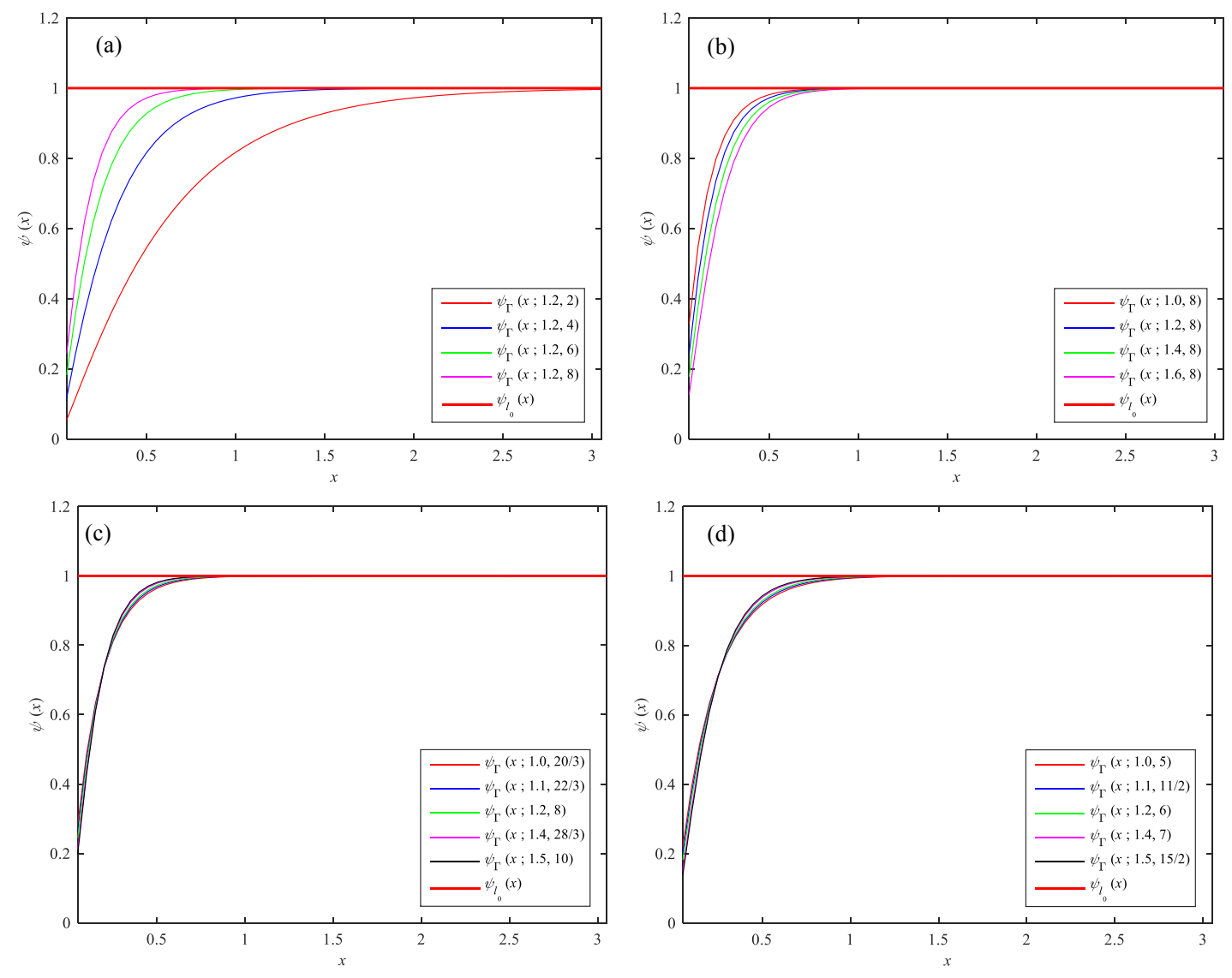


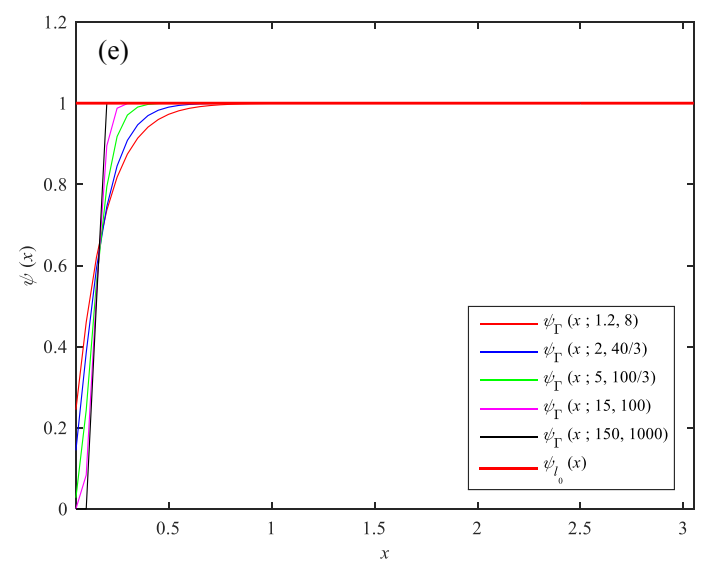

Fig. 2: The plots of the CDF of Gamma distribution $\psi_{\mathrm{r}}(x ; \alpha, \beta)$ with different shape parameter $\alpha$ and scale parameter $\beta$.

\section{Experiments}

The performance of $\Gamma_{a}$-WLS and $\Gamma_{i}$-WLS was tested on two simulated numeric 2-D phantoms and the Catphan 600 physical phantom. Our experiments include the comparison with the methods $l_{2}$-WLS, $l_{1 a}$-WLS and $l_{1 i}$-WLS. The abbreviations and their full names are listed in Table 1 . The maximum iteration number $N_{0}$ was fixed to 500 in all the reconstructions, and $\varepsilon_{0}$ was set to $1 \times 10^{-7}$ and $1 \times 10^{-8}$ in the simulated and Catphan phantom, respectively. The shape parameter $\alpha$ was set to 1.2, and the scale parameter $\beta$ to the value calculated according to the above parameter strategy. The balance parameter $\lambda$ was adjusted to give the best visual effect in all the reconstructions.

Table 1. Different methods used in experiment with the abbreviations.

Table 1. Different methods used in experiment with the abbreviations.
\begin{tabular}{|c|c|}
\hline Abbreviation & Reconstruction methods \\
\hline$l_{2}$-WLS & $l_{2}$-norm regularization with weighted least square (Eq. (12)) \\
\hline$l_{1 a}$-WLS & $l_{1}$-norm regularization with anisotropic weighted least square (Eq. (13)) \\
\hline$l_{1 i}$-WLS & $l_{1}$-norm regularization with isotropic weighted least square (Eq. (14)) \\
\hline$\Gamma_{a}$-WLS & Gamma regularization with anisotropic weighted least square (Eq. (19)) \\
\hline$\Gamma_{i}$-WLS & Gamma regularization with isotropic weighted least square (Eq. (21)) \\
\hline
\end{tabular}

\subsection{Simulated numeric phantoms experiments}

The two simulated phantoms contain the Modified Shepp-Logan (MSL) phantom (Fig. 3 (a)) and a simulated non-uniform rational B-splines (NURBS) based cardiac-torso (NCAT) phantom (Fig. 3 (b)) (Segars and Tsui 2002). These phantoms are composed by $256 \times 256$ pixels with $1 \mathrm{~mm} \times 1 \mathrm{~mm}$ pixel size. A monochromatic CT parallel scanner with a total of 180 scanning views and 367 radial bins per view is modeled. We simulated two CT dose levels by adding noisel and noise 2 according to Eq. (2) and (3) with parameters $T=10000, h=5$ and $T=10000, h=10$ (Lu et al 2001, Li et al 2004), respectively. For convenience, the corresponding low dose sinograms are named M-sin1(sinogram of MSL phantom with added noise1), M-sin2 (sinogram of MSL phantom with added noise2), N-sin1 (sinogram of NACT phantom with noise1) and N-sin2 (sinogram of NACT phantom with noise2), respectively. All the simulated sinograms are illustrated in Fig. 4. Quantitative assessments are given in terms of PSNR (peak signal to noise ratio), SNR (signal to noise ratio), and SSIM (structural Similarity Index measuring) (Wang et al 2004). These quantitative metrics are defined by Eq. (33)-(35):

$$
\begin{gathered}
\operatorname{PSNR}(P, I)=10 \log _{10}\left(\frac{P_{\max }^{2}}{\frac{1}{m n} \sum_{i=1}^{m} \sum_{j=1}^{n}\left(P_{i, j}-I_{i, j}\right)^{2}}\right) \\
\operatorname{SNR}(P, I)=10 \log _{10}\left(\frac{\sum_{i=1}^{m} \sum_{j=1}^{n}\left(P_{i, j}\right)^{2}}{\sum_{i=1}^{m} \sum_{j=1}^{n}\left(P_{i, j}-I_{i, j}\right)^{2}}\right)
\end{gathered}
$$




$$
\operatorname{SSIM}(P, I)=\frac{1}{m n} \frac{\left(2 \mu_{p} \mu_{i}+C_{1}\right)\left(2 \sigma_{p i}+C_{2}\right)}{\left(\mu_{p}^{2}+\mu_{i}^{2}+C_{1}\right)\left(\sigma_{p}^{2}+\sigma_{i}^{2}+C_{2}\right)}
$$

where $I$ is the reconstructed image and $P$, the ground-truth image. $P_{\max }$ denotes the maximum intensity value in $P . \mu_{p}$ and $\mu_{i}$ are the mean values of the $8 \times 8$ square window in $I$ and $P$. $\sigma_{p}$ and $\sigma_{i}$ are the corresponding standard deviations, and $\sigma_{p i}$ is the corresponding covariance. $C_{1}, C_{2}$ and $C_{3}$ are three constant parameters, which are set according to (Wang et al 2004). $C_{1}=(0.01 \times L)^{2}, C_{2}=(0.03 \times L)^{2}, C_{3}=C_{2} / 2$, with $L$ denoting the grayscale range of the image to reconstruct.
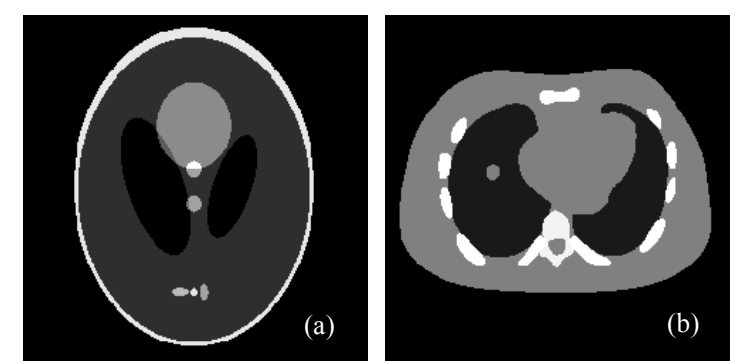

Fig. 3: The phantoms used in this paper. (a) MSL phantom, (b) NACT phantom
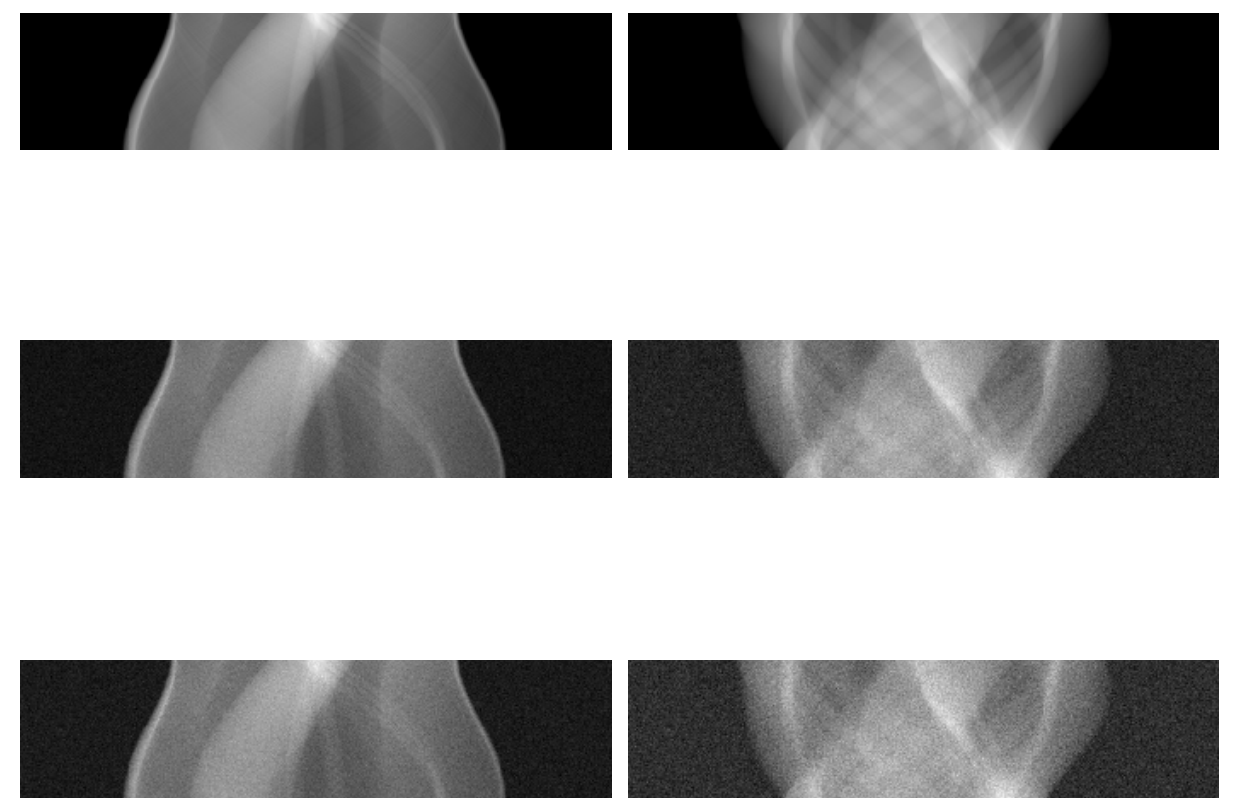

Fig. 4: Simulated projection data. (a): the simulated sinogram of MSL phantom; (a1)-(a2): the simulated low dose sinograms $\mathrm{M}-\sin 1$ and M-sin2 for MSL phantom; (b): the simulated sinogram of NACT phantom; (b1-b2): the simulated low dose sinograms $\mathrm{N}-\sin 1$ and $\mathrm{N}-\sin 2$ for NACT phantom.

The reconstruction results from the proposed approaches were compared with the results obtained from the $l_{2}$-WLS, $l_{1 a}$-WLS and $l_{1 i}$-WLS methods. In the experiments conducted on the MSL phantom, with the shape parameter $\alpha$ set to 1.2, the scale parameter $\beta$ was calculated to 6.0 and 4.2 for the $\Gamma_{a}$-WLS and $\Gamma_{i}$-WLS methods, respectively. In the experiments performed on the NACT phantom, with the shape parameter $\alpha$ set to 1.2 , the scale parameter $\beta$ was calculated to 16.7 and 11.8 for the $\Gamma_{a}$-WLS and $\Gamma_{i}$-WLS methods, respectively. Fig. 5 gathers the reconstructed images for all the simulations carried out with $\mathrm{M}-\sin 1, \mathrm{M}-\sin 2, \mathrm{~N}-\sin 1$ and $\mathrm{N}-\sin 2$, Fig. 5 (a1-d1), (a2-d2), (a3-d3),

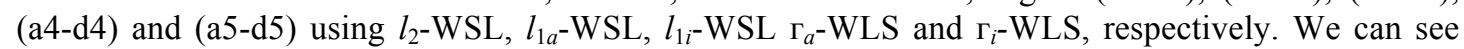
that the proposed $\Gamma_{a}$-WLS and $\Gamma_{i}$-WLS methods perform better in preserving edges and suppressing noise than other methods. Table 2 and Table 3 list the PSNR, SNR and SSIM values of the 
reconstructed images with respect to the reference phantom images. The $\lambda$ values are also listed in Table 2 and Table 3. We can note that the $\Gamma_{a}$-WLS and $\Gamma_{i}$-WLS approaches lead to reconstructions with higher PSNR, SNR and SSIM values. However, the computation time required by the proposed algorithm is higher than for the other methods as shown in Table 2 and Table 3. Fig. 7 depicts the intensity profiles (specified as the red lines in Fig.6) for the reconstructions in Fig.5. A better match with the ground truth phantom profiles can be observed for the $\Gamma_{a}$-WLS and $\Gamma_{i}$-WLS methods. We can note that, though giving higher PSNR values than the $\Gamma_{a}$-WLS algorithm, the $\Gamma_{i}$-WLS algorithm can reconstruct images with no significant visual difference with respect to the results from the $\Gamma_{a}$-WLS algorithm.
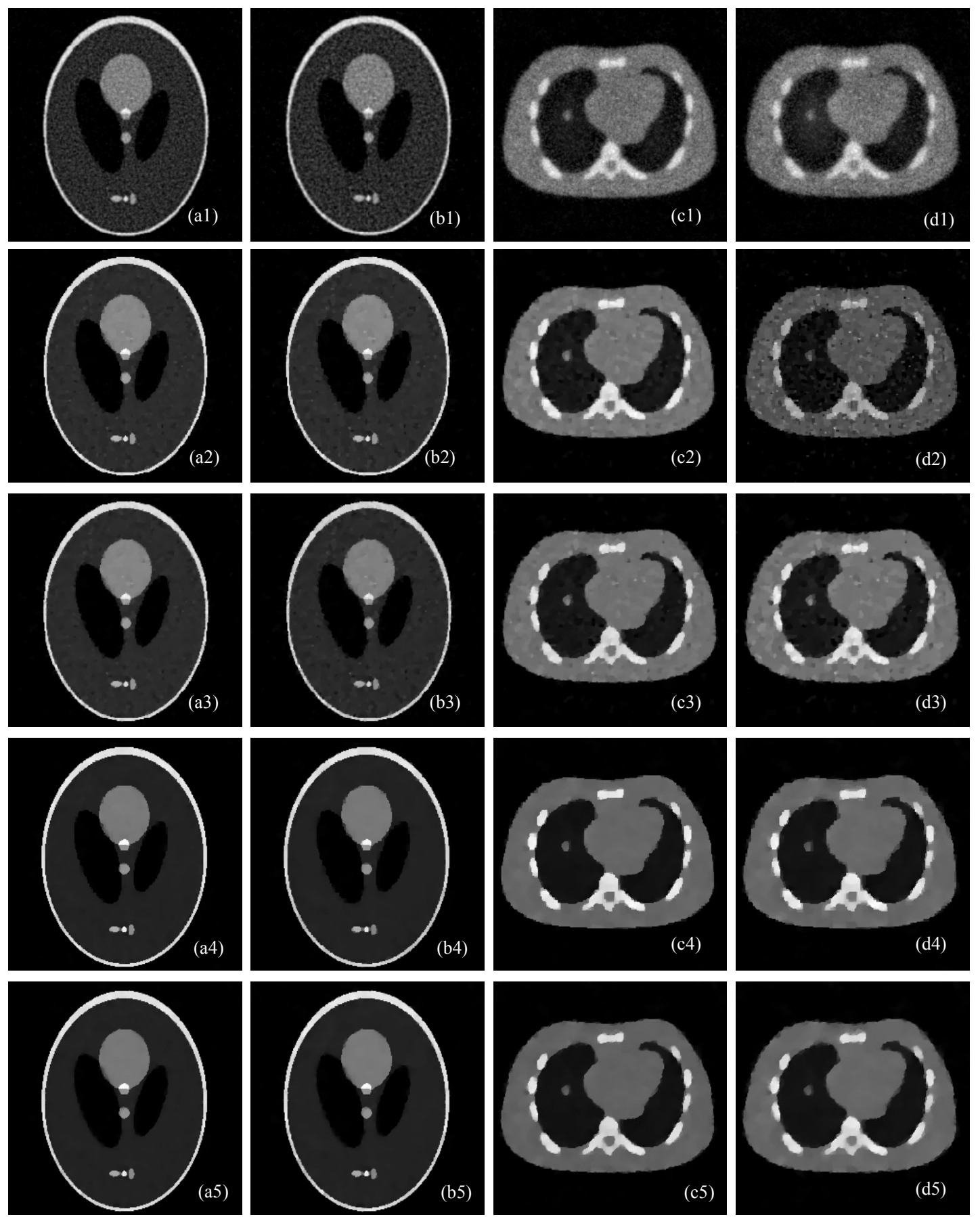

Fig. 5: Reconstructed images from the sinograms $\mathrm{M}-\sin 1, \mathrm{M}-\sin 2, \mathrm{~N}-\sin 1$ and $\mathrm{N}-\sin 2$, respectively. Columns from left to right correspond to the results reconstructed from $\mathrm{M}-\sin 1, \mathrm{M}-\sin 2, \mathrm{~N}-\sin 1$ and $\mathrm{N}-\sin 2$, respectively. Rows from top to bottom correspond to the images reconstructed using the methods of $l_{2}$-WSL, $l_{1 a}-\mathrm{WSL}, l_{1 i}-\mathrm{WSL}, \Gamma_{a}-\mathrm{WLS}$ and $\Gamma_{i}$-WLS, respectively. 
Table 2: Quantitative evaluation of the reconstructed MSL phantom images for the different methods.

\begin{tabular}{|c|c|c|c|c|c|c|c|c|c|c|}
\hline \multirow{3}{*}{ Method } & \multicolumn{10}{|c|}{ MSL phantom } \\
\hline & \multicolumn{5}{|c|}{$M-\sin 1$} & \multicolumn{5}{|c|}{$M-\sin 2$} \\
\hline & $\lambda$ & PSNR & SNR & SSIM & $\begin{array}{l}\text { Iterations/ Time } \\
\text { (in minutes) }\end{array}$ & $\lambda$ & PSNR & SNR & SSIM & $\begin{array}{l}\text { Iterations/ Time } \\
\text { (in minutes) }\end{array}$ \\
\hline$l_{2}-\mathrm{WSL}$ & 6.8 & 24.48 & 12.37 & 0.60 & $127 / 0.96$ & 5.6 & 23.81 & 11.69 & 0.62 & $269 / 1.88$ \\
\hline$l_{1 a}-\mathrm{WSL}$ & 2.4 & 28.42 & 17.01 & 0.84 & $283 / 2.00$ & 2.3 & 28.07 & 15.96 & 0.84 & $283 / 2.01$ \\
\hline$l_{1 i}-\mathrm{WSL}$ & 4.6 & 28.71 & 16.60 & 0.93 & $170 / 1.43$ & 3.3 & 27.73 & 15.62 & 0.90 & $176 / 1.33$ \\
\hline$\Gamma_{a}-\mathrm{WLS}$ & 2.5 & 31.55 & 19.43 & 0.96 & $234 / 2.30$ & 2.0 & 28.33 & 16.22 & 0.95 & $201 / 1.97$ \\
\hline$\Gamma_{i}$-WLS & 4.4 & 32.03 & 19.92 & 0.97 & $262 / 2.32$ & 3.0 & 29.19 & 17.07 & 0.95 & $271 / 2.35$ \\
\hline
\end{tabular}

Table 3: Quantitative evaluation of the reconstructed NACT phantom images for different methods.

\begin{tabular}{|c|c|c|c|c|c|c|c|c|c|c|}
\hline \multirow{3}{*}{ Method } & \multicolumn{10}{|c|}{ NACT phantom } \\
\hline & \multicolumn{5}{|c|}{$\mathrm{N}-\sin 1$} & \multicolumn{5}{|c|}{$\mathrm{N}-\sin 2$} \\
\hline & $\lambda$ & PSNR & SNR & SSIM & $\begin{array}{l}\text { Iterations/ Time } \\
\text { (in minutes) }\end{array}$ & $\lambda$ & PSNR & SNR & SSIM & $\begin{array}{c}\text { Iterations/ Time } \\
\text { (in minutes) }\end{array}$ \\
\hline$l_{2}-\mathrm{WSL}$ & 9.4 & 22.07 & 12.59 & 0.65 & $20 / 0.92$ & 9.6 & 20.08 & 10.60 & 0.63 & $281 / 2.91$ \\
\hline$l_{1 a}-\mathrm{WSL}$ & 3.2 & 24.94 & 15.47 & 0.87 & $355 / 0.87$ & 3.3 & 24.76 & 15.29 & 0.89 & $79 / 0.82$ \\
\hline$l_{1 i}$-WSL & 5.5 & 26.44 & 16.97 & 0.93 & $101 / 0.87$ & 4.0 & 24.91 & 15.44 & 0.90 & $50 / 0.64$ \\
\hline$\Gamma_{a}-\mathrm{WLS}$ & 1.0 & 26.98 & 17.50 & 0.96 & $164 / 1.81$ & 1.0 & 25.64 & 16.17 & 0.94 & $164 / 1.71$ \\
\hline$\Gamma_{i}-\mathrm{WLS}$ & 1.6 & 27.49 & 18.01 & 0.96 & $140 / 1.38$ & 1.2 & 25.92 & 16.44 & 0.94 & $171 / 1.63$ \\
\hline
\end{tabular}
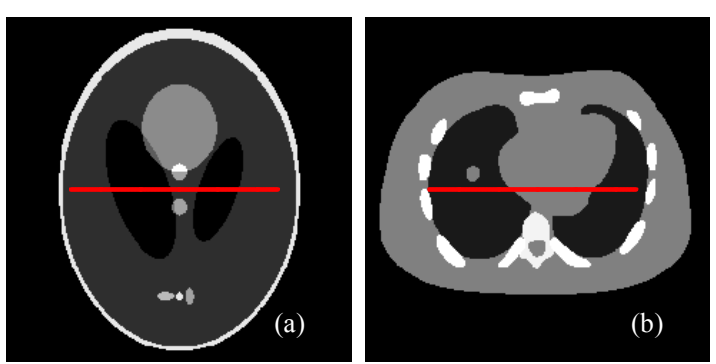

Fig. 6: The profiles (the $128^{\text {th }}$ row from the top) of the two phantoms
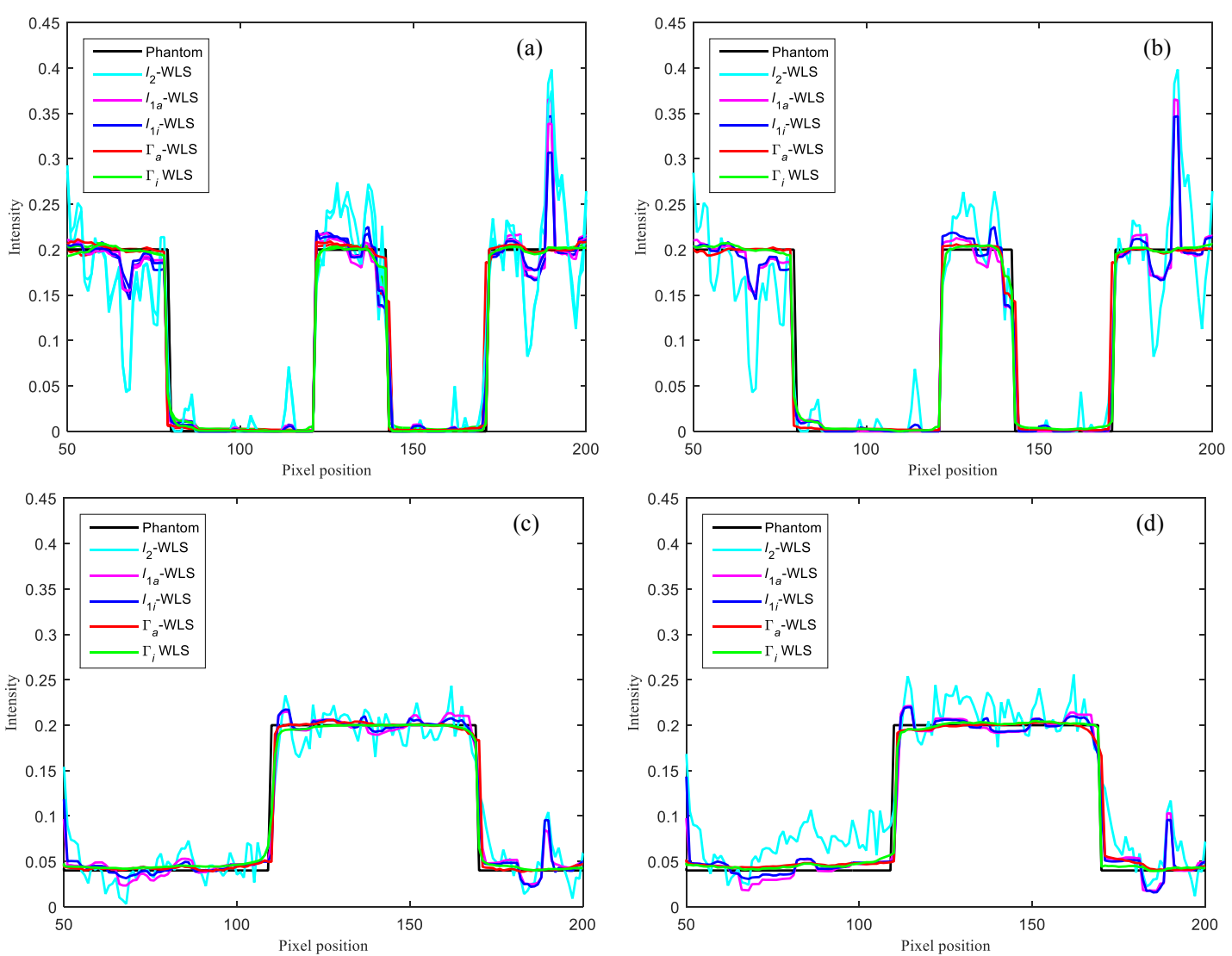

Fig. 7: Depicts of the horizontal profiles (the $128^{\text {th }}$ row, as shown in Fig. 6) of the phantoms images reconstructed by different methods. (a)-(b) are the horizontal profiles of images reconstructed from M-sin1 and M-sin2, respectively; (c)-(d) are the horizontal profiles of images reconstructed from $\mathrm{N}-\sin 1$ and $\mathrm{N}-\sin 2$, respectively. 


\subsection{Catphan 600 data experiments}

Experiments on the Catphan 600 phantom under low dose protocol were also performed to evaluate the algorithm performance on the preservation of high resolution and low contrast features. The tube voltage and the current were set to $100 \mathrm{KVp}$ and $10 \mathrm{~mA}$. The images to reconstruct are composed by $256 \times 256$ pixels with $1 \mathrm{~mm} \times 1 \mathrm{~mm}$ pixel size. The imaging geometry was specified as: the source to isocenter distance was $500 \mathrm{~mm}$ and the distance from the source to the detector was set to $1500 \mathrm{~mm}$. The data were acquired from a monochromatic $\mathrm{CT}$ fan beam scanner with a total of 661 scanning views and 1024 radial bins per view. In the experiments worked out using the Catphan 528 phantom, with the shape parameter $\alpha$ set to 1.2 , the scale parameter $\beta$ was calculated based on the above parameter setting strategy as 550.46 and 322.58 for $\Gamma_{a}$-WLS and $\Gamma_{i}$-WLS, respectively. For the Catphan 404, $\alpha$ being still set to 1.2 , following the same procedure, the scale parameter $\beta$ values were respectively calculated to be 419.58 and 402.68 . The global parameter $\lambda$ was set to give the best results in terms of edge preservation and noise suppression.

The reconstructions of the central 2-D slices in phantoms Catphan 528 and Catphan 404 are displayed in Fig. 8 and Fig. 9, respectively. In Fig. 8 and Fig. 9, the reconstructed images (a)-(f) correspond to the algorithms FBP (with ramp filter), $l_{2}$-WSL, $l_{1 a}$-WSL, $l_{1 i}$-WSL, $\Gamma_{a}-\mathrm{WLS}$ and $\Gamma_{i}$-WLS, respectively. We can see that the images reconstructed by FBP suffer from severe noise, and the $l_{2}$-WSL algorithm leads to noise suppression at the cost of obvious detail blurring. The $\Gamma_{a}$-WLS and $\Gamma_{i}$-WLS methods perform better in noise suppression and edge preservation than the others. From Fig 8 (c)-(f), it is found that the proposed methods provide reconstructions with higher resolution than the $l_{1}$-norm regularization methods (see the structures pointed out by red arrows in Fig. 8). In Fig.(9), we can also observe that the proposed methods can preserve low contrast regions with clearer boundaries than the $l_{1}$-norm regularization based methods in Fig.8 (c) and Fig.8 (d) (see the structures defined by red arrows in Fig. 9).
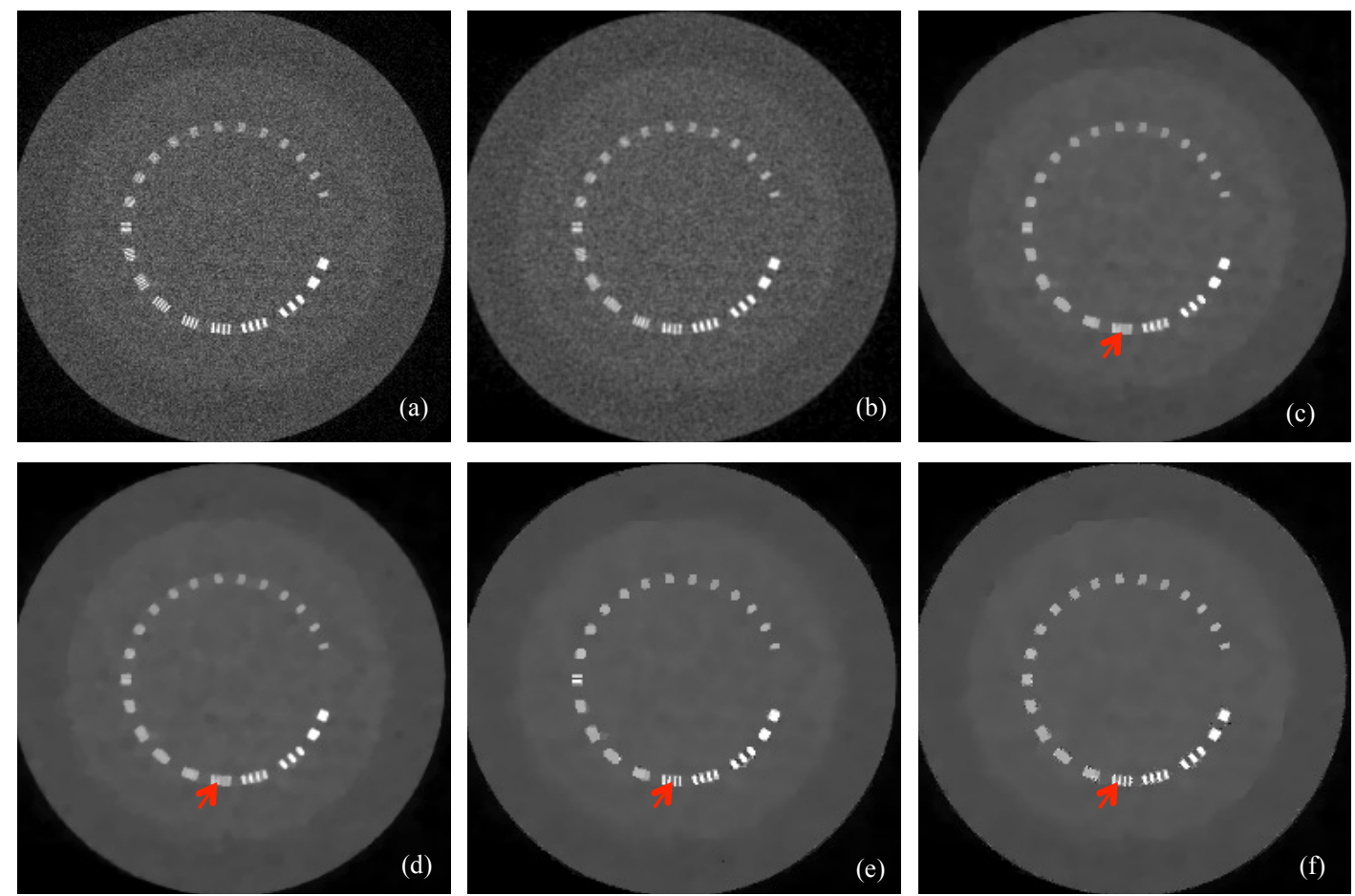

Fig. 8: Reconstruction results of the central 2-D slice in phantom Catphan 528. (a) the image reconstructed by FBP (ramp filter). (b) the image reconstructed by the $l_{2}$-WSL method $(\lambda=20)$, (c) the image reconstructed by the $l_{1 a}$-WSL method $(\lambda=0.4)$, (d) he image reconstructed by the $l_{1 i}$-WSL method $(\lambda=0.4)$, (e) the image reconstructed by the $\Gamma_{a}$-WLS method $(\lambda=0.0061)$, (f) the image reconstructed by the $\Gamma_{i}$-WLS method $(\lambda=0.0121)$. 

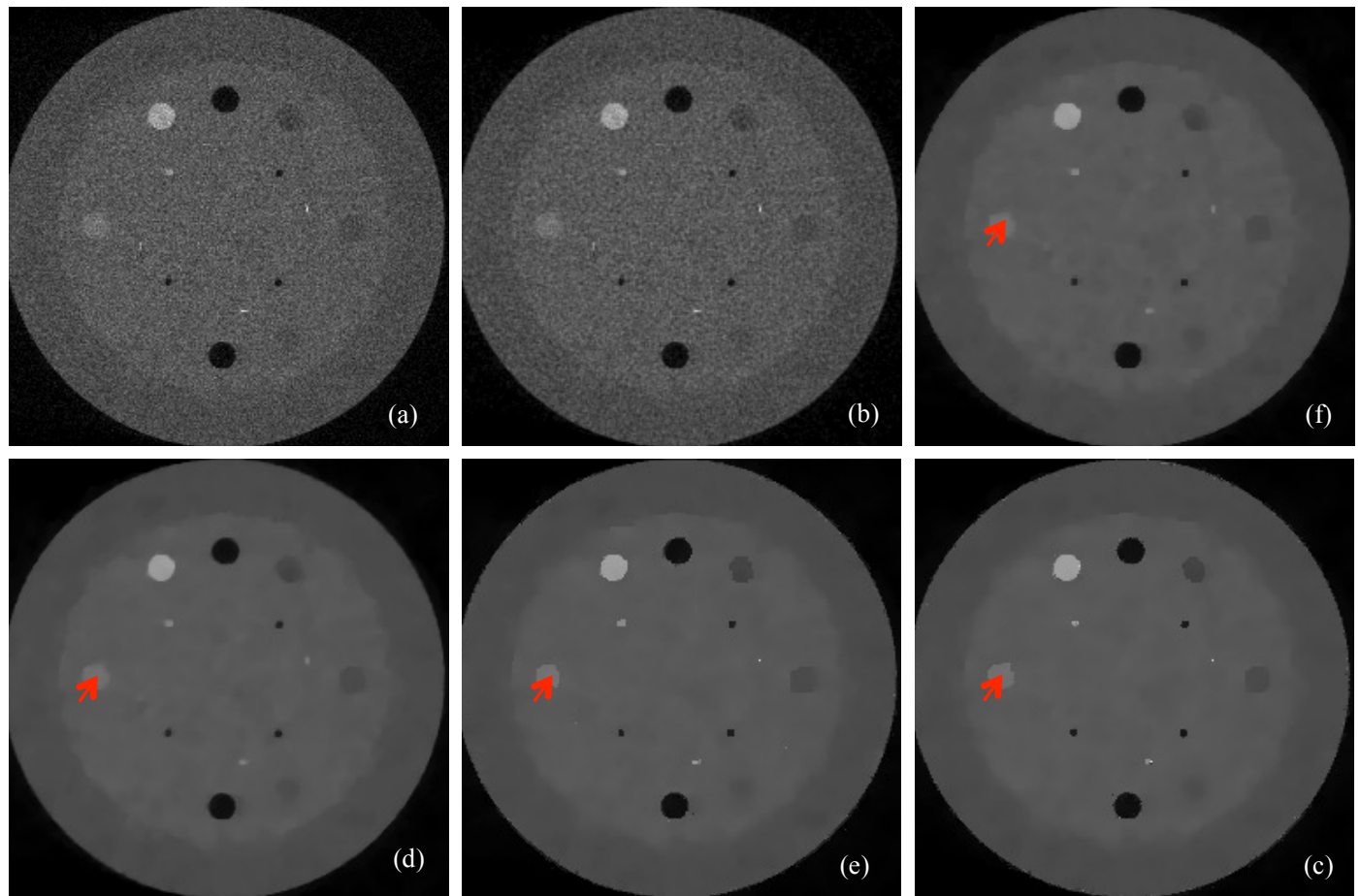

Fig. 9: Reconstruction results of the central 2-D slice in phantom Catphan 404. (a) the image reconstructed by FBP (ramp). (b) the image reconstructed by the $l_{2}$-WSL method $(\lambda=10)$, (c) the image reconstructed by the $l_{1 a}$-WSL method $(\lambda=0.4)$, (d) the image reconstructed by the $l_{1 i}$-WSL method $(\lambda=0.3)$, (e) the image reconstructed by the $\Gamma_{a}$-WLS method $(\lambda=0.009)$, (f) the image reconstructed by the $\Gamma_{i}$-WLS method $(\lambda=0.011)$.

\subsection{Convergence}

The convergence property of the $\Gamma_{a}$-WLS and $\Gamma_{i}$-WLS algorithms is analyzed based on the cost functions in Eq. (19) and Eq. (21). M-sin1 was used for this analysis. The evolutions of the cost function values and the PSNR values over iterations are depicted in Fig. 10. We can observe monotonic decrease of the log-transformed cost function values and the monotonic increase of the PSNR values (of reconstructed images) as the iteration proceeds.
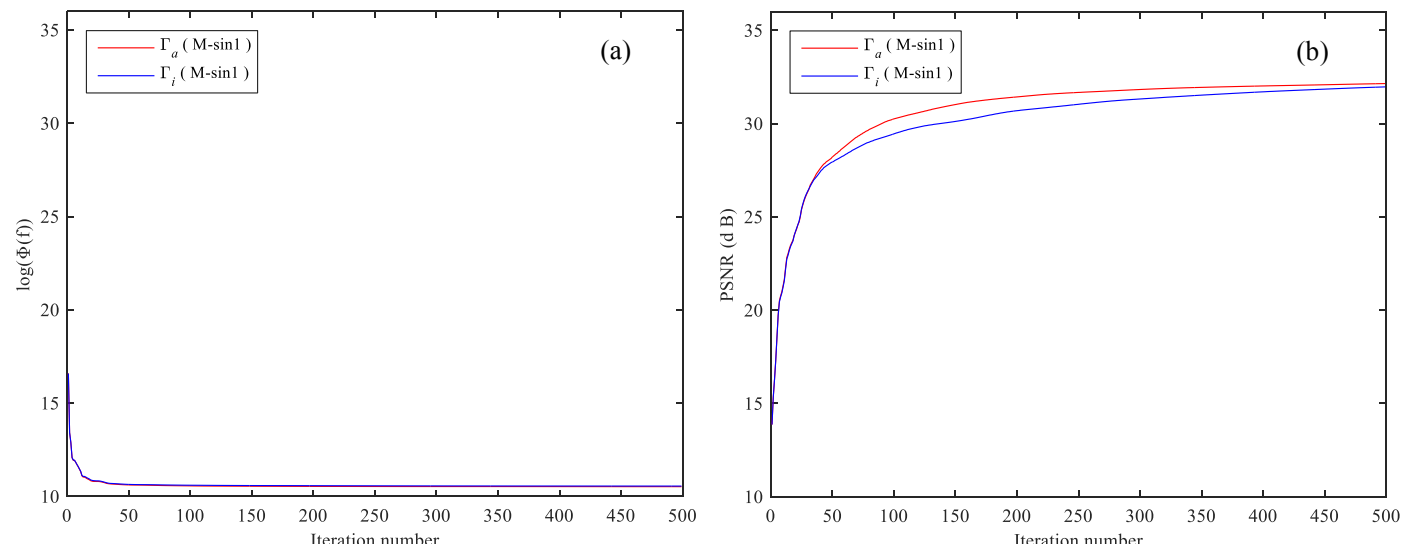

Fig. 10: (a) and (b) show the values of cost function and PSNR versus iterations for the MSL phantom data with $\Gamma_{a}-$ WLS and $\Gamma_{i}$-WLS algorithms, respectively.

\subsection{Sensitivity analysis of the shape parameter $\alpha$}

Only the shape parameter $\alpha$ needs to be set in the proposed parameter setting strategy. Fig. 11 shows the reconstructed images with $\mathrm{M}$-sin1 and $\mathrm{N}-\sin 1$ using $\Gamma_{a}$-WLS and $\Gamma_{i}$-WLS when the shape parameter $\alpha$ is set to 1.2, 1.8, 2.0 and 5.0, and the parameter $\beta$ calculated as $\alpha / E$ following the above parameter setting strategy. Fig. 11 confirms the proposed parameter setting strategy and that the choice of $\alpha$ equal to 1.2 leads to reconstructed images with higher quality than others. In particular, as $\alpha$ is increased more noise from small gradients can be observed in the reconstructed images. 


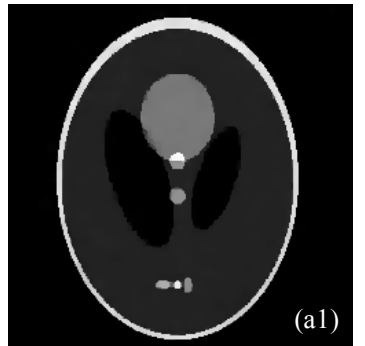

$\lambda=3.1, \alpha=1.2, \beta=6$

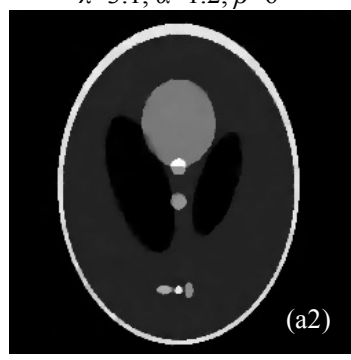

$\lambda=4.7, \alpha=1.2, \beta=4.2$

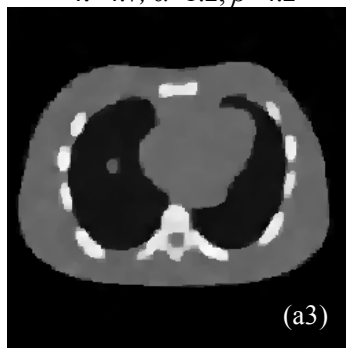

$\lambda=1.1, \alpha=1.2, \beta=16.7$

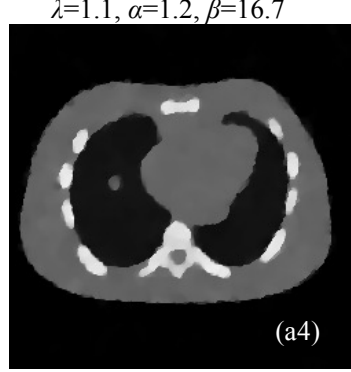

$\lambda=1.8, \alpha=1.2, \beta=11.8$

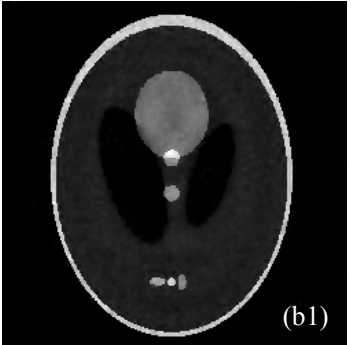

$\lambda=1.9, \alpha=1.8, \beta=9$

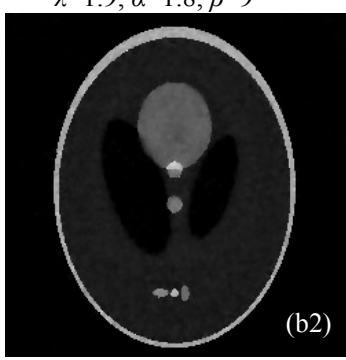

$\lambda=3.2, \alpha=1.8, \beta=6.4$

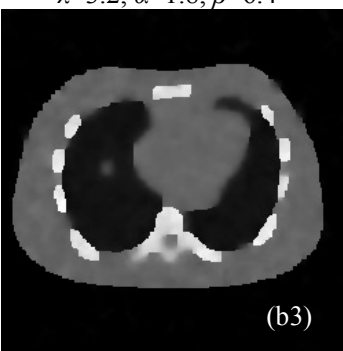

$\lambda=3.2, \alpha=1.8, \beta=25$

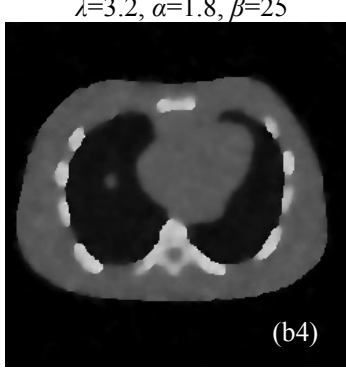

$\lambda=3.5, \alpha=1.8, \beta=17.7$

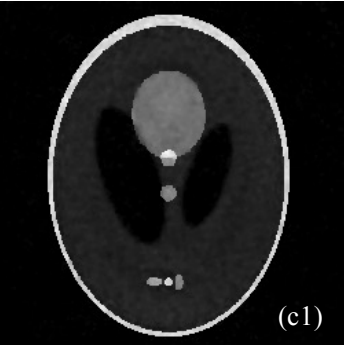

$\lambda=2.6, \alpha=2, \beta=10$

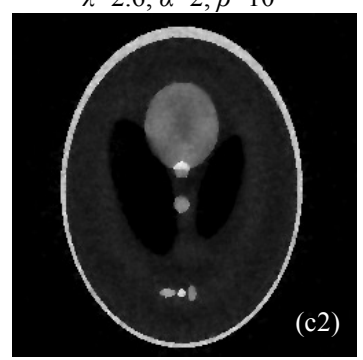

$\lambda=3.3, \alpha=2, \beta=7.1$

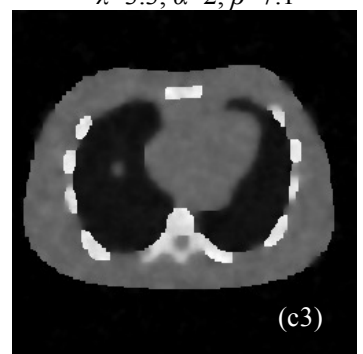

$\lambda=3.3, \alpha=2, \beta=27.8$

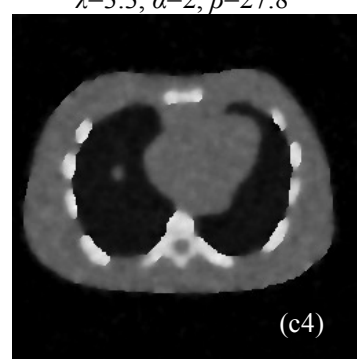

$\lambda=3.3, \alpha=2, \beta=19.6$

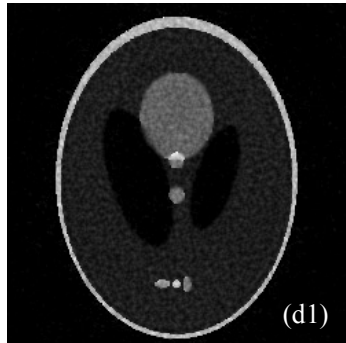

$\lambda=1.3, \alpha=5, \beta=25$

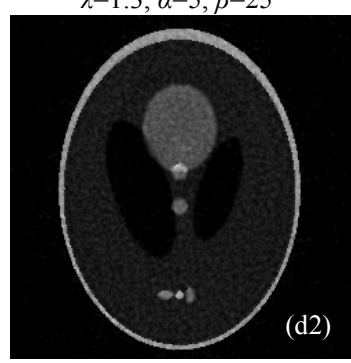

$\lambda=2.5, \alpha=5, \beta=17.7$

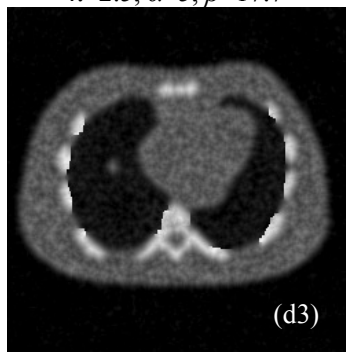

$\lambda=2.5, \alpha=5, \beta=69.4$

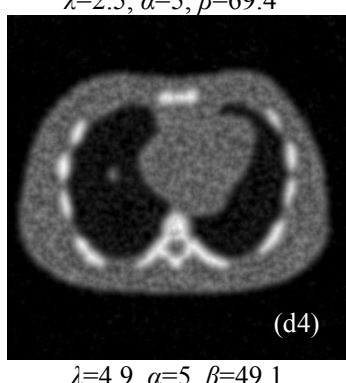

Fig. 11: (a1-d1): The reconstructed images by $\Gamma_{a}$-WLS using M-sin1; (a2-d2): The reconstructed images by $\Gamma_{i}$-WLS using M-sin1; (a3-d3): The reconstructed images by $\Gamma_{a}-$ WLS using N-sin1; (a4-d4): The reconstructed images by $\Gamma_{a}$-WLS using N-sin1.

\subsection{Comparison with another $l_{0}$-approximate regularization with tunable parameter}

In this section, we compared our method with another $l_{0}$-approximate regularization with tunable parameter, which was proposed by $\mathrm{Hu}$ et al (Hu et al 2011). This regularization also uses a tunable parameter $p$ to realize an approximation to the $l_{0}$ norm regularization, with the regularization function given by Eq. (36):

$$
\psi_{\mathrm{Hu}}(x ; p)=\log \left(\frac{x}{p}+1\right)
$$

Here, $x$ denotes image gradient. The regularization function $\psi_{\mathrm{Hu}}$ modulates the function by changing the scale through the parameter $p$. We can see in Fig. 12 (a) that the regularization function $\psi_{\mathrm{Hu}}$ exhibits a worse approximation to $l_{0}$ norm regularization.

We also provide the reconstructions of M-sin 1 and N-sin 1 employing our methods $\left(\Gamma_{a}-\mathrm{WLS}\right.$ and $\Gamma_{i}$-WLS) and the regularization function $\psi_{\mathrm{Hu}}$ with the same weighted least square models used in above experiments. The methods using the regularization function $\psi_{\mathrm{Hu}}$ are referred as $\mathrm{H}_{a}$-WLS and $\mathrm{H}_{i}$-WLS for the anisotropic and isotropic models, respectively. The reconstruction results are illustrated in Fig. 13 with different combinations of parameters $p$ and $\lambda$ given below. We can see that the proposed Gamma regularization leads to reconstruction results with better performance in both edge preservation and noise suppression than the reconstructions with $\mathrm{H}_{a}-\mathrm{WLS}$ and $\mathrm{H}_{i}$-WLS. 

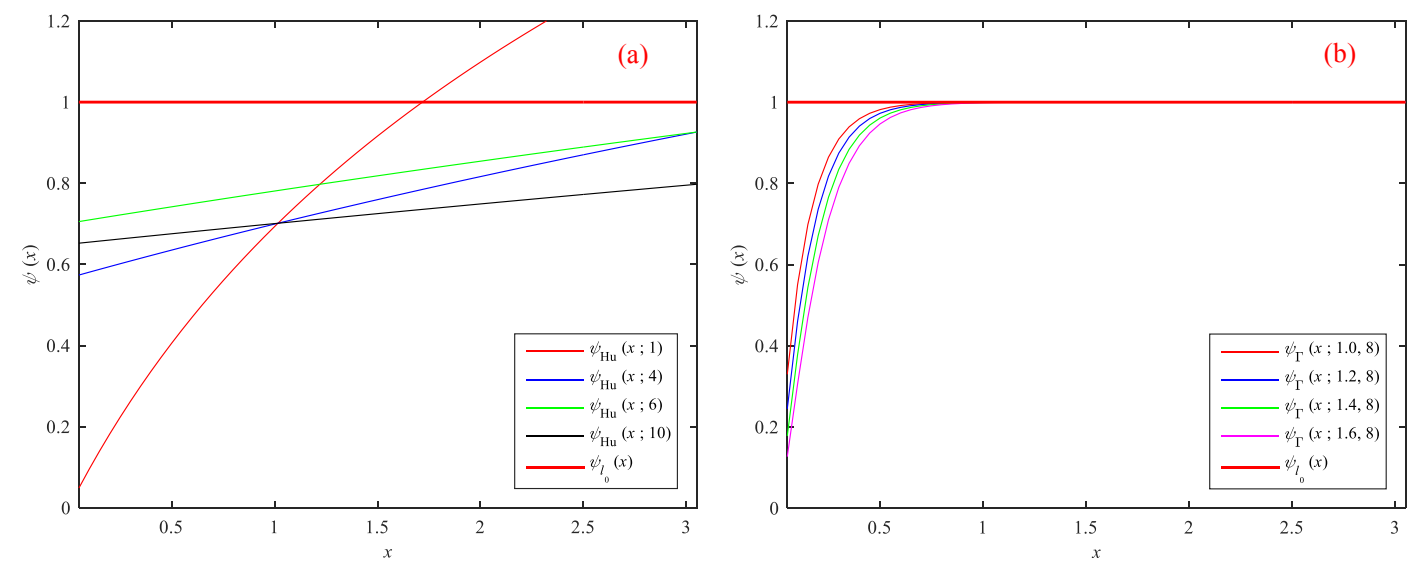

Fig. 12: (a) Regularization function $\psi_{\text {Ни }}(x ; p)$ of Hu method (Hu et al 2011) with different tunable parameter $p$. (b) Gamma regularization function $\psi_{\mathrm{r}}(x ; \alpha, \beta)$ with different parameters for the proposed regularization.

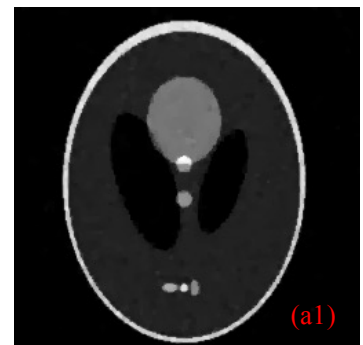

$\lambda=4.5, p=1$

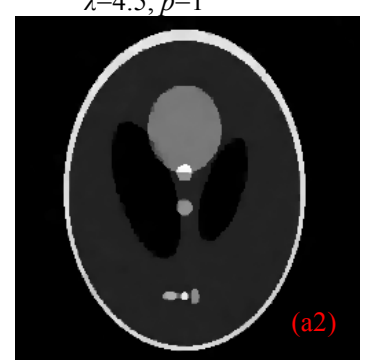

$\lambda=3.1, \alpha=1.2, \beta=6$

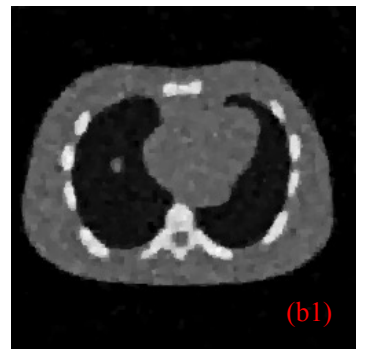

$\lambda=4.7, p=1$

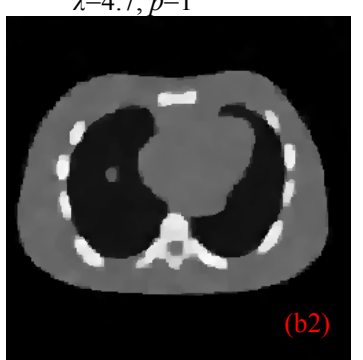

$\lambda=1.1, \alpha=1.2, \beta=16.7$

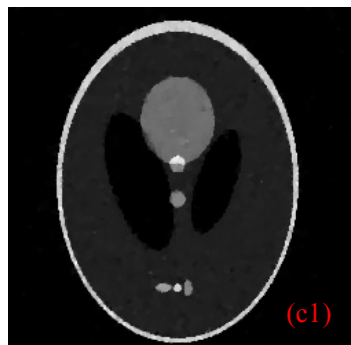

$\lambda=4.2, p=1$

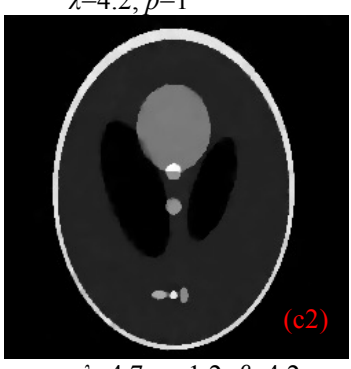

$\lambda=4.7, \alpha=1.2, \beta=4.2$

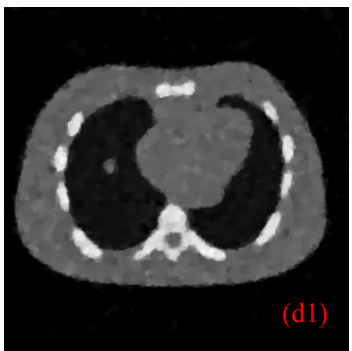

$\lambda=4.8, p=1$

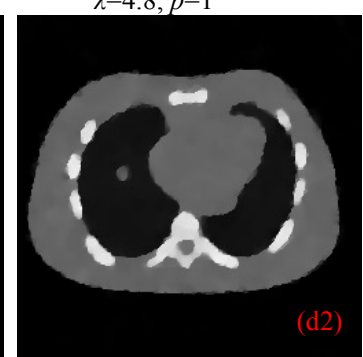

$\lambda=1.8, \alpha=1.2, \beta=11.8$

Fig. 13: The reconstructed images of MSL phantom data for the approaches $H_{a}-W L S, H_{i}-$ WLS and $\Gamma_{a}$-WLS and $\Gamma_{i}$-WLS. (a1)-(b1): the reconstruction results with $\mathrm{H}_{a}$-WLS. (c1)-(d1): the reconstruction results with $\mathrm{H}_{i}$-WLS. (a2-b2): the reconstruction results with $\Gamma_{a}$-WLS. (c2-d2): the reconstruction results with $\Gamma_{i}$-WLS.

\section{Conclusion}

In this paper, we described two iterative reconstruction algorithms $\Gamma_{a}$-WLS and $\Gamma_{i}$-WLS for LDCT image reconstruction based on a Gamma regularization and we compared them to several well-known schemes with integer norms. Both simulated data and Catphan 600 data were used to test the proposed methods. From the experiments, we have seen that the proposed Gamma regularizations have better performance in edge preservation and noise suppression than other methods. Nevertheless, practical application of the proposed algorithms still needs further validation using more clinical data. From the Table 2 and Table 3, we found that their computation times are higher than other methods, and some acceleration techniques should be applied to increase its feasibility.

The proposed Gamma regularization realizes the rendering of a flexible regularization effect via modulating the shape parameter $\alpha$ and the scale parameter $\beta$. In this study, the shape parameter was set to 1.2 to obtain a regularization function adapted to the variations of the image gradients, and the value $\beta$ was selected based on the preset ratio $E$ with $5 E$ equal to about $25 \%$ quantile of the gradient amplitude ensemble. Though this parameter setting strategy is proved to be effective in the phantom experiments conducted so far, a thorough analysis on parameter sensitivity is still required through its application to clinical data. 


\section{Acknowledgements}

We are indebted to the reviewers for their worthy comments that allowed major improvements of this paper. We also thank Dr. Jianhua Ma in Southern Medical University for providing the low dose CT projection data. This research was supported by National Basic Research Program of China under grant (2010CB732503), National Natural Science Foundation under grant (81370040), and by the Qing Lan Project in Jiangsu Province.

\section{References}

Armijo L 1966 Minimization of functions having Lipschitz continuous first partial derivatives Pacific J. Math 16 1-3

Beck A and Teboulle M 2009 Fast gradient-based algorithms for constrained total variation image denoising and deblurring problems IEEE Trans. Image Process. 18 2419-2434

Boyd S, Vandenberghe L 2004 Convex Optimization (Cambridge University Press)

Brenner D J, Elliston C D, Hall E J and Berdon W E 2001 Estimated risks of radiation-induced fatal cancer from pediatric CT Am J Roentgenol. 176 289-296

Brenner D J and Hall E J 2007 Computed tomography_an increasing source of radiation exposure N. Engl. J. Med. 357 2277-2284

Candes E, Romberg J, and Tao T 2006a Robust uncertainty principles: Exact signal reconstruction from highly incomplete frequency information IEEE Trans. Inf. Theory $\mathbf{5 2}$ 489-509

Candes E, Romberg J, and Tao T 2006b Stable signal recovery from incomplete and inaccurate measurements Commun. Pure Appl. Math. 59 1207-1223

Chen Y, Shi L Y, Feng Q J, Yang J, Shu H Z, Luo L M, Coatrieux J L, Chen W F 2014 Artifact suppressed dictionary learning for low dose CT image processing IEEE Trans. Med. Imaging 33 2271-2292

Chen Y, Yin X D, Shi L Y, Feng Q J, Yang J, Shu H Z, Luo L M, Coatrieux J L and Chen W F 2013 Improving abdomen tumor low-dose CT images using a fast dictionary learning based processing Phys. Med. Biol. 58 5803-5820

Chen Z Q, Jin X, Li L and Wang G 2013 A limited-angle CT reconstruction method based on anisotropic TV minimization Phys. Med. Biol. 58 2119-2141

de González A B and Darby S 2004 Risk of cancer from diagnostic X-rays: estimates for the UK and 14 other countries Lancet $363345-351$

Donoho D, 2006 Compressed sensing IEEE Trans. Inf. Theory 52 1289-1306

Elbakri I A and Fessler J A 2002 Statistical image reconstruction for polyenergetic X-ray computed tomography IEEE Trans. Med. Imaging. $2188-99$

Fang L Y, Li S T, McNabb R P, Nie Q, Kuo A N, Toth C A, Izatt J A and Farsiu S 2013 Fast Acquisition and Reconstruction of Optical Coherence Tomography Images via Sparse Representation IEEE Trans. Med. Imaging $322034-2049$

Fletcher R and Reeves C 1964 Function minimization by conjugate gradients Comput. J. 7 149-154

Frank M and Wolfe P 1956 An algorithm for quadratic programming Naval Research Logistics Quarterly $395-110$

Guo W H and Yin W T 2012 Edge guided reconstruction for compressive imaging SIAM J. Imaging. Sci. 5 809-834

Gonzalez R C, Woods R E and Eddins S L 2004 Digital Image Processing using MATLAB (Pearson Education)

Hogg R V and Craig A T 1978 Introduction to Mathematical Statistics, 4th edition (New York: Macmillan)

Hsieh J 1998 Adaptive streak artifact reduction in computed tomography resulting from excessive X-ray photon noise Med. Phys. 25 2139-2147

Hsieh J 2009 Computed Tomography Principles, Design, Artifacts, and Recent Advances (Bellingham, WA: SPIE Optical Engineering Press)

Hu Y N, Xie L Z, Luo L M, Nunes J C and Toumoulin C. 2011 L0 constrained sparse reconstruction for multi-slice helical CT reconstruction Phys. Med. Biol. 56 1173-1189

Joseph P M 1982 An improved algorithm for reprojecting rays through pixel images IEEE Trans. Med. Imaging1192-196

Lange K and Carson R 1984 EM reconstruction algorithms for emission and transmission tomography J. Comput. Assist. Tomogr. 8 306-316

Li T F, Li X, Wang J, Wen J H, Lu H B and Liang Z R 2004 Nonlinear sinogram smoothing for low-dose X-ray CT IEEE Trans. Nucl. Sci. $512505-2513$

Liu Y, Liang Z R, Ma J H, Lu H B, Wang K, Zhang and Moore H W 2014 Total Variation-Stokes Sparse-View X-ray CT Image Reconstruction IEEE Trans. Med. Imaging 33 749-763

Lu H B, Hsiao I T, Li X, Liang Z G 2001 Noise properties of low-dose CT projections and noise treatment by scale transformations," in Proc. IEEE NSS-MIC Conf. Rec. 3 1662-1666

Lu Y J, Zhang X Q, Douraghy A, Stout D, Tian J, Chan T F and Chatziioannou A F 2009 Source Reconstruction for Spectrally-resolved Bioluminescence Tomography with Sparse A priori Information Opt. Express 17 8062-8080

Lu Y, Zhao J and Wang G 2012 Few-view image reconstruction with dual dictionary Phys. Med. Biol. 57 173-189

Lustig M, Donoho D, and Pauly J M 2007 Sparse MRI: The application of compressed sensing for rapid MR imaging Magnetic Resonance in Medicine $\mathbf{5 8} 1182-1195$

Man B D and Basu S 2004 Distance-driven projection and backprojection in three dimensions," Phys. Med. Biol. 49 2463-2475

Niu S Z, Gao Y, Bian Z Y, Hang J, Chen W F, Liang Z R and Ma J H 2014 Sparse-view x-ray CT reconstruction via total Generalized variation regularization Phys. Med. Biol. 59 2997-3017

Peters T M 1981 Algorithms for fast back-and re-projection in computed tomography IEEE Trans. Nucl. Sci. 28 $3641-3647$

R. Hyndman and Y. Fan 1996 Sample quantiles in statistical packages American Statistician (American Statistical Association) 50. 361-365

Rudin L I, Osher S and Fatemi E 1992 Nonlinear total variation based noise removal algorithms Physica D. 60 259-268

Segars W P and Tsui B M W 2002 Study of the efficacy of respiratory gating in myocardial SPECT using the new 4-D NCAT phantom IEEE Trans. Nucl. Sci. 49 675-679

Sidky E Y, Kao C M, and Pan X 2006 Accurate image reconstruction from few-views and limited-angle data in divergent-beam CT J. X-Ray Sci. Technol. 14 119-139 
Siddon R 1985 Fast calculation of the exact radiological path of a three-dimensional CT array Med. Phys. 12 252-255

Tikhonov A N and Arsenin V Y 1997 Solution of Ill-Posed Problem (New York: Winston)

Wang J, Li T, Lu H and Liang Z 2006 Penalized weighted least-squares approach to sinogram noise reduction and image reconstruction for low-dose x-ray CT IEEE Trans. Med. Imaging 25 1272-1283

Wang Z, Bovik A C, Sheikh H R, and Simoncelli E P 2004 Image Quality Assessment: From Error Visibility to Structural Similarity IEEE Trans. Image Process. 13 600- 612

Xu Q, Yu H Y, Mou X Q, Zhang L, Hsieh J and G. Wang 2012 Low- dose X-ray CT Reconstruction via Dictionary Learning IEEE Trans. Medical Imaging 31 1682-1697

Yu H Y and Wang G 2010 A soft-threshold filtering approach for reconstruction from a limited number of projections Phys. Med. Biol. 55 3905-3916

Zhuang W, Gopal S S, Hebert T J 1994 Numerical evaluation of methods for computing tomographic projections IEEE Trans. Nucl. Sci. 41 1660-1665 